August 2004 - NREL/SR-510-36392

\title{
Kinetic and Modeling Investigation to Provide Design Guidelines for the NREL Dilute- Acid Process Aimed at Total Hydrolysis/Fractionation of Lignocellulosic Biomass
}

July 1998

Y.Y. Lee, P. lyer, Q. Xiang, and J. Hayes Auburn University

Auburn, Alabama
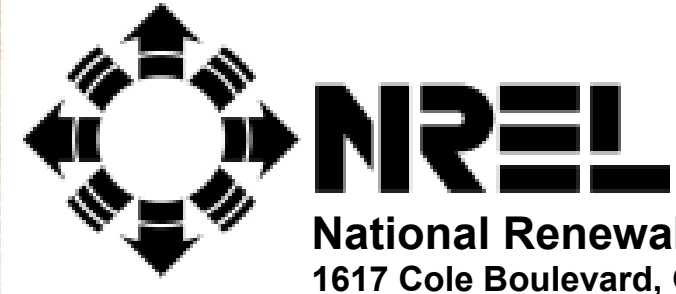

National Renewable Energy Laboratory 1617 Cole Boulevard, Golden, Colorado 80401-3393 303-275-3000 • www.nrel.gov

Operated for the U.S. Department of Energy Office of Energy Efficiency and Renewable Energy by Midwest Research Institute • Battelle 
NREL Technical Monitor: R. Torget

Prepared under Subcontract No. RCG-7-17041-01

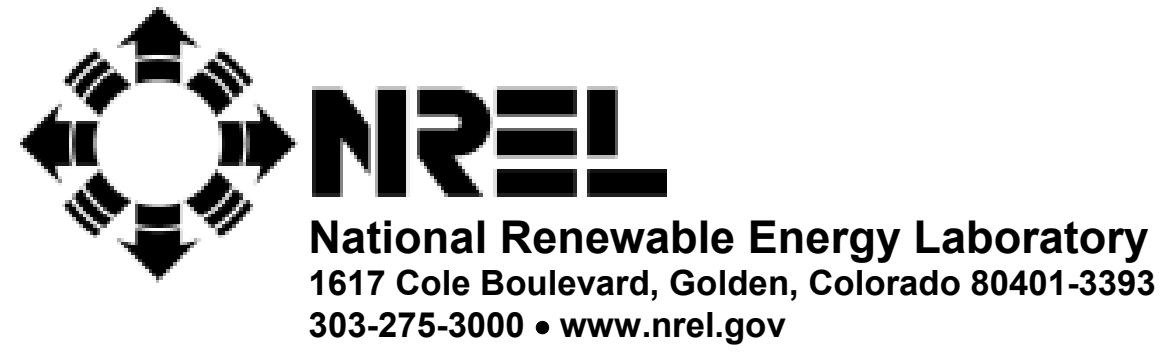

Operated for the U.S. Department of Energy

Office of Energy Efficiency and Renewable Energy

by Midwest Research Institute • Battelle

Contract No. DE-AC36-99-GO10337 
This publication was reproduced from the best available copy Submitted by the subcontractor and received no editorial review at NREL

\section{NOTICE}

This report was prepared as an account of work sponsored by an agency of the United States government. Neither the United States government nor any agency thereof, nor any of their employees, makes any warranty, express or implied, or assumes any legal liability or responsibility for the accuracy, completeness, or usefulness of any information, apparatus, product, or process disclosed, or represents that its use would not infringe privately owned rights. Reference herein to any specific commercial product, process, or service by trade name, trademark, manufacturer, or otherwise does not necessarily constitute or imply its endorsement, recommendation, or favoring by the United States government or any agency thereof. The views and opinions of authors expressed herein do not necessarily state or reflect those of the United States government or any agency thereof.

Available electronically at http://www.osti.gov/bridge

Available for a processing fee to U.S. Department of Energy and its contractors, in paper, from:

U.S. Department of Energy

Office of Scientific and Technical Information

P.O. Box 62

Oak Ridge, TN 37831-0062

phone: 865.576.8401

fax: 865.576 .5728

email: mailto:reports@adonis.osti.gov

Available for sale to the public, in paper, from:

U.S. Department of Commerce

National Technical Information Service

5285 Port Royal Road

Springfield, VA 22161

phone: 800.553 .6847

fax: 703.605.6900

email: orders@ntis.fedworld.gov

online ordering: http://www.ntis.gov/ordering.htm 
TABLE OF CONTENTS

TASK 1. Modeling of Countercurrent Shrinking-Bed Reactor in Dilute-Acid Total-Hydrolysis of Lignocellulosic Biomass

Model development......................................................

Mathematical Modeling...............................................

Dispersion Model Simulation Results and Discussion.....................8

TASK 2. Verification of Hydrolysis Kinetics of Cellulose of Yellow Poplar Sawdust using 0.07wt\% Sulfuric Acid

Experiment...............................................................

Data Analysis........................................................

TASK 3. Preliminary Investigation on Thermal/Oxidative Cracking of Lignin Generated from Hydrolysis Process

Oxidative Degradation of Lignin by Hydrogen Peroxide.....................37

Experimental........................................................... 38

Direction of Future Research...........................................42

Preparation of Lignin Generated during Total Hydrolysis of Yellow Poplar

Appendix 1. Fortran Program Source Codes for Modeling of Counter-Current Shrinking-Bed Reactor

Appendix2. List of Papers Published or Submitted for Publication 


\section{Task 1: Modeling of Countercurrent Shrinking-Bed Reactor in Dilute-Acid Total- Hydrolysis of Ligoncellulosic Biomass}

\section{Model Development}

The kinetic pattern of dilute-acid hydrolysis of lignocellulosic materials and the conceptual sketch of counter-current reactor are shown in Figure 1. The countercurrent reactor is simply a continuous flow reactor in which the directions of liquid and solid are reversed. The shrinking-bed operation in the countercurrent reactor induces continual reduction of solid velocity moving through the reactor. Hemicellulose and cellulose exhibit the biphasic kinetic behavior upon acid hydrolysis. In Fig.1, the fast-hydrolyzed fraction of hemicellulose is represented by $\mathrm{H}_{1}$, and the slow-hydrolyzed fraction as $\mathrm{H}_{2}$. The two hemicelluloses are first hydrolyzed to form soluble oligomers, then the oligomers are converted to xylose and decomposed products. However, in the case of cellulose, the hydrolysis of cellulose is often modeled simply as two consecutive reactions as shown in Fig. 1. This is because, the amorphous cellulose which is taken to be the fast-hydrolyzed portion, proceeds extremely fast and also no oligomeric concentration is generally detected. A universal model is developed which is applicable to both hemicellulose and cellulose hydrolysis and also for shrinking and nonshrinking bed operation by appropriate adjustment of process parameters. An important feature of this new model is that axial dispersion (or back mixing) is considered in the liquid flow.

\section{Mathematical Modeling}

Previous investigations indicate that the acid hydrolysis of lignocellulosic biomass can be modeled as first-order reactions. The rate constant, $\mathrm{k}_{\mathrm{i}}$, is then represented by the Arrhenius equation in which the acid concentration term, $\mathrm{S}$, is absorbed into the pre-exponential factor. 


$$
k_{i}=k_{i o} S^{n i} e^{-E_{i} / R T}
$$

A mathematical model for the dilute-acid hydrolysis of hemicellulose is first developed as follows. The following assumptions are made in this modeling: (1) axial dispersion (or backmixing) for solid flow is negligible; (2) the reaction and physical conditions (temperature, acid concentration, porosity, solid bulk density, and bed voidage) are uniform within the reactor; (3) the internal and external mass transfer effects are negligible.

Because the first order nature and the isothermal condition, the hemicellulose hydrolysis kinetic pattern shown in Figure 1 can be simplified into unbranched reaction sequence:

$$
\mathrm{H} \stackrel{\mathrm{K}_{\mathrm{H}}}{\longrightarrow} \mathrm{O} \stackrel{\mathrm{k}_{3}}{\longrightarrow} \mathrm{X} \stackrel{\mathrm{k}_{4}}{\longrightarrow} \mathrm{D}
$$

Where $\mathrm{K}_{\mathrm{H}}=\mathrm{F}^{*} \mathrm{k}_{1}+(1-\mathrm{F})^{*} \mathrm{k}_{2}$, and $\mathrm{F}$ is the fraction of easily hydrolyzable hemicellulose.

An overall solid material balance and a material balance for hemicellulose $(H)$ within a differential segment of the reactor under steady state give the following differential equations.

$$
\begin{gathered}
\rho(1-\varepsilon) \frac{d v}{d x}-\mathrm{fK}_{H} C_{H}=0 \\
\frac{d\left(\mathrm{vC}_{H}\right)}{d x}-K_{H} C_{H}=0
\end{gathered}
$$

With the boundary conditions of:

$$
\begin{array}{ll}
\mathrm{x}=\mathrm{L}, & \mathrm{v}=\mathrm{v}_{\mathrm{O}} \\
\mathrm{x}=\mathrm{L}, & \mathrm{C}_{\mathrm{H}}=\mathrm{CHO}_{\mathrm{H}}
\end{array}
$$

Where $\varepsilon$ is the bed voidage; $\rho$ is the bulk density of feedstock; and $f$ is the ratio of total mass solubilized over hemicellulose solubilized during the hydrolysis. The factor, $f$, is an entity greater than one since components other than hemicellulose are also dissolved during the process including lignin, extraneous components, and small fraction of cellulose. The hemicellulose 
content in the solid $\left(\mathrm{C}_{\mathrm{H}}\right)$ is defined on the basis of the reactor volume. It is notable that $\mathrm{v}$ (the solid linear velocity) is treated as a variable (not a constant) in the bed shrinking model. One would expect that $\mathrm{v}$ decreases as the solid moves through the reactor, the effect of shrinking.

From Equations 2 and 3, the following single equation is obtained.

$$
\mathrm{v} \frac{\mathrm{dC}_{\mathrm{H}}}{\mathrm{dx}}+\left[\mathrm{fk}_{\mathrm{H}} / \rho(1-\varepsilon)\right] \mathrm{C}_{\mathrm{H}}^{2}-\mathrm{k}_{\mathrm{H}} \mathrm{C}_{\mathrm{H}}=0
$$

With the boundary condition of:

$$
\mathrm{x}=\mathrm{L}, \quad \mathrm{C}_{\mathrm{H}}=\mathrm{CHO}_{\mathrm{H}}
$$

From the material balance for oligomer $(\mathrm{O})$ and xylose $(\mathrm{X})$ in the liquid stream, the following additional set of differential equations is obtained.

$$
\begin{aligned}
& D \frac{d^{2} C_{o}}{d x^{2}}-u \frac{d C_{0}}{d x}+k_{t}\left(C_{H} / \varepsilon\right)-k_{3} C_{o}=0 \\
& D \frac{d^{2} C_{x}}{d x^{2}}-u \frac{d C_{x}}{d x}+k_{3} C_{o}-k_{4} C_{x}=0
\end{aligned}
$$

With the boundary conditions of:

$$
\begin{array}{ll}
\mathrm{x}=0, & \mathrm{C}_{\mathrm{O}}=0 \\
\mathrm{x}=0, & \mathrm{C}_{\mathrm{X}}=0 \\
\mathrm{x}=\mathrm{L}, & \frac{\mathrm{dC}_{\mathrm{o}}}{\mathrm{dx}}=0 \\
\mathrm{x}=\mathrm{L}, & \frac{\mathrm{dC}_{\mathrm{x}}}{\mathrm{dx}}=0
\end{array}
$$

The concentration terms, $\mathrm{C}_{0}$ and $\mathrm{C}_{\mathrm{X}}$, are defined on the basis of liquid volume.

In dimensionless form, Equations (4)-(6) are expressed as:

$$
\begin{aligned}
& \frac{d Y_{H}}{d Z}+\frac{\beta \omega}{q}\left[\left(Y_{H}^{2} / \lambda\right)-Y_{H}\right]=0 \\
& (1 / P e) \frac{d^{2} Y_{O}}{d Z^{2}}-\frac{d Y_{O}}{d Z}+(\beta / \varepsilon) Y_{H}-\beta \alpha_{3} Y_{O}=0
\end{aligned}
$$




$$
(1 / P e) \frac{d^{2} Y_{X}}{d Z^{2}}-\frac{d Y_{X}}{d Z}+\beta \alpha_{3} Y_{O}-\beta \alpha_{4} Y_{X}=0
$$

where $\mathrm{q}=\mathrm{v} / \mathrm{v}_{\mathrm{O}}, \beta=\mathrm{k}_{\mathrm{H}} \mathrm{L} / \mathrm{u}, \omega=\mathrm{u} / \mathrm{v}_{\mathrm{O}}, \lambda=(1-\varepsilon) \rho /\left(\mathrm{C}_{\mathrm{HO}} \mathrm{f}\right), \mathrm{Z}=\mathrm{x} / \mathrm{L}, \mathrm{Y}_{\mathrm{H}}=\mathrm{C}_{\mathrm{H}} / \mathrm{C}_{\mathrm{HO}}$,

$$
\mathrm{Y}_{\mathrm{O}}=\mathrm{C}_{\mathrm{O}} / \mathrm{C}_{\mathrm{HO}}, \mathrm{Y}_{\mathrm{X}}=\mathrm{C}_{\mathrm{x}} / \mathrm{C}_{\mathrm{HO}}, \mathrm{Pe}=\mathrm{Lu} / \mathrm{D}, \alpha_{3}=\mathrm{k}_{3} / \mathrm{k}_{\mathrm{H}}, \alpha_{4}=\mathrm{k}_{4} / \mathrm{k}_{\mathrm{H}}
$$

With boundary conditions of:

$$
\begin{array}{ll}
\mathrm{Z}=1, & Y_{\mathrm{H}}=1 \\
\mathrm{Z}=0, & Y_{\mathrm{O}}=0 \\
\mathrm{Z}=1, & \frac{\mathrm{dY}_{\mathrm{O}}}{\mathrm{dZ}}=0 \\
\mathrm{Z}=0, & \mathrm{Y}_{\mathrm{X}}=0 \\
\mathrm{Z}=1, & \frac{\mathrm{dY_{X }}}{\mathrm{dZ}}=0
\end{array}
$$

The initial hemicellulose concentration in the solid feedstock is defined as

$$
\mathrm{C}_{\mathrm{HO}}=\mathrm{H}_{\mathrm{O}}(1-\varepsilon) \rho \mathrm{v}_{\mathrm{O}}
$$

Where $\mathrm{H}_{\mathrm{O}}$ is the initial hemicellulose content in untreated biomass. The yield is defined

as

$$
\text { Yield }=\frac{(\text { xlose }+ \text { oligomer) recovered in liquid }}{\text { Total hemicellulose content in solid feedstock }}=\frac{\varepsilon u\left(C_{0}+C_{x}\right)}{v_{O} C_{H O}}=\varepsilon \omega\left(Y_{O}+Y_{x}\right)
$$

\section{Shrinking Factor, q}

A shrinking factor $(q)$ is defined as the ratio of solid linear velocity (v) at any point in the reactor to that at the reactor entrance point $\left(\mathrm{v}_{\mathrm{O}}\right)$. It is an entity that is less than one since bed shrinking reduces the solid linear velocity. An explicit solution can be obtained for the shrinking factor from Equations (2) and (3). In the process one needs to express $\mathrm{dv} / \mathrm{dx}$ term explicitly on the left had side in Equation (2) and likewise for $\mathrm{d}\left(\mathrm{vC}_{\mathrm{H}}\right) / \mathrm{dx}$ in Equation (3) and dividing one equation by the other. The resulting equation is then integrated to obtain the solution for $\mathrm{q}$ as 
expressed by Equation (10). It is seen that the shrinking factor is a function of hemicellulose composition in solid $\left(\mathrm{Y}_{\mathrm{H}}\right)$ only since $\lambda$ is a constant $\left(\lambda=\rho / \mathrm{fC}_{\mathrm{HO}}\right)$.

$$
\mathrm{q}=\frac{\mathrm{v}}{\mathrm{v}_{\mathrm{O}}}=\frac{\lambda-1}{\lambda-\mathrm{Y}_{\mathrm{H}}}
$$

Equation 7 can be simplified using Equation 10 to give the following

$$
\frac{d Y_{H}}{d Z}-\frac{\beta \omega}{\lambda(\lambda-1)}\left[Y_{H}^{3}-2 \lambda Y_{H}^{2}+\lambda^{2} Y_{H}\right]=0
$$

Equations 8,9 and 11 will be solved simultaneously with aforementioned boundary conditions and using kinetic parameters selected from previous investigations (Table 1). The important process variables such as solid feeding velocity, linear liquid velocity, temperature, bed shrinking, and sulfuric acid concentration will be studied. Sugar concentration, yield and reactor processing capacity are output parameters which will be used to assess reactor performance.

An important feature of the model for hemicellulose is that it is also applicable to cellulose hydrolysis as well as the non-shrinking bed operation with the adjustment of process parameters. For example, with the assumption of $\mathrm{k}_{1}=\mathrm{k}_{2}=\mathrm{k}_{\mathrm{C}}$ and $\mathrm{k}_{3}=\mathrm{k}_{\mathrm{G}}, \mathrm{k}_{4}=0$ the hemicellulose kinetic pattern is simplified into two consecutive first-order reactions which represents the pattern for the cellulose hydrolysis. The conversion of the model from hemicellulose into cellulose becomes complete with adjustment of the kinetic parameters $\left(\mathrm{E}_{\mathrm{i}}, \mathrm{k}_{\mathrm{i} 0}\right.$, and $\left.\mathrm{n}_{\mathrm{i} 0}\right)$. Furthermore, if one arbitrarily take the $f$ value to be very small, then $\lambda$ value becomes very large and $q$ value approaches 1 indicating no changes in solid velocity. The model under this special case then becomes a non-shrinking one. 


\section{Dispersion Model Simulation Results and Discussion}

Yellow Poplar Sawdust was selected as the substrate in this study. On dry basis, it was analyzed to contain $19.63 \%$ XMG [xylose (15.48)+mannose(3.08)+galactose $(1.08)], 47.98 \%$ glucan, $22.33 \% \mathrm{~K}$. lignin, and $10.06 \%$ others. Based on our discussion with NREL, regarding very dilute-acid hydrolysis on yellow poplar, it was assumed that $50 \%$ of the lignin and others were also solubilized along with hemicellulose. Therefore the $f$ value is calculated to be 1.82 during the stage of hemicellulose hydrolysis. After the hemicellulose fraction is completely solubilized, the remaining solid is calculated to contain $85 \%$ glucan and a total of $15 \%$ lignin and other materials. It was also assumed that $50 \%$ of lignin and others are solubilized along with cellulose hydrolysis. The value of $\mathrm{f}$ is therefore calculated to be 1.09 in the subsequent cellulose hydrolysis.

The kinetic parameters used in this study are selected from previous investigations (Table

1). The following known and estimated process parameters are introduced for the simulation.

(1) About $6 \mathrm{~g}$ of substrate can be packed in a $30 \mathrm{~mL}$ reactor which gives $\rho^{*}(1-\epsilon)=0.2$ and it was assumed constant throughout regardless of the solubilization of hemicellulose and cellulose ( our lab data)

(2) The fraction of fast-hydrolyzed portion of the total hemicellulose of yellow poplar (F) is 0.70 . (our previous investigation)

(3) The length of the reactor is 6" (actual size of our laboratory reactor).

(4) Peclet number, a measure of axial backmixing was assumed equal to 20. 
The important process variables studied in this work are the solid feeding velocity, linear liquid velocity, temperature, bed shrinking, and sulfuric acid concentration. Sugar concentration, yields, and high reactor processing capacities are the output parameters considered in connection with the reactor performance.

\section{Effect of Initial Solid Feeding Rate}

The effect of the initial solid feeding rate was investigated at $190^{\circ} \mathrm{C}$ with $0.08 \mathrm{wt} \%$ sulfuric acid and the liquid linear velocity of $2.0 \mathrm{~cm} / \mathrm{min}$. The initial solid feeding rate is an index for the reactor processing capacity. The basic reaction conditions are selected from previous investigations. As shown in Figure 2 the hemicellulose content in solid and solid linear velocity are the function of the initial solid feeding rate $\left(\mathrm{v}_{\mathrm{o}}\right)$ and the reactor position $(\mathrm{Z})$. The shape of the hemicellulose profiles indicates that the solid feeding rate $\left(\mathrm{v}_{\mathrm{o}}\right)$ is an important factor affecting the reactor performance and thus needs to be optimized. Taking the profile of hemicellulose where $\mathrm{v}_{\mathrm{o}}=5.0 \mathrm{~cm} / \mathrm{min}$ in Fig.2, it is clear that the conversion is too low, achieving only $53.3 \%$ at the solid exit point $(Z=0)$. Taking the other extreme where $\mathrm{v}_{\mathrm{O}}=0.5 \mathrm{~cm} / \mathrm{min}$, the hydrolysis reaction is now overly done as the conversion is essentially complete where $Z=0.56$, or within the first $44 \%$ of the reactor path (note that $Z=1$ at the solid entering point). The optimum of $\mathrm{v}_{0}\left(\mathrm{~V}_{\mathrm{o}}\right.$, op $)$ does exist. The true optimum can only be determined from an economic model accounting the yield, product concentration and processing capacity among other things. In this work we take a simplistic approach by setting an arbitrary criterion that $\mathrm{v}_{0}$ reaches optimum when the conversion for hemicellulose at the solid exit point $(\mathrm{Z}=0)$ is 0.995 or $\mathrm{Y}_{\mathrm{A}}=0.5 \%$. Under the given set of conditions, the optimum initial solid feeding rate is found to be $1.09 \mathrm{~cm} / \mathrm{min}$. At a rate lower than this, the hydrolysis of hemicellulose is finished far before the outlet point causing excessive 
decomposition. At a rate higher than the optimum, the hydrolysis of hemicellulose is incomplete leaving much of the hemicellulose unreacted when the solid leaves the reactor.

It is also seen in Figure 2 that both hemciellulose content in solid and the solid linear velocity decline rather abruptly then level off along the direction of solid feeding. With $\mathrm{v}_{\mathrm{O}}=$ $1.09 \mathrm{~cm} / \mathrm{min}, 90 \%$ of original hemicellulose is hydrolyzed when the solid is at the half way point in the reactor $(\mathrm{Z}=0.5)$. This means that the major fraction of sugar is produced in vicinity of the solid inlet point and it has relatively a small distance to travel before being washed out the reactor. It is the primary feature of the countercurrent reactor that minimizes the residence time for sugar degradation thus raising the yield and sugar concentration. This feature is further enhanced in the shrinking-bed operation. In this mode, the solid moving velocity slows down as the reaction proceeds. The solid with unhydrolyzed hemicellulose stays even to the liquid outlet point.

The modeling results are similar for cellulose hydrolysis (Figure 3). The optimum initial solid feeding rate was determined to be $0.6 \mathrm{~cm} / \mathrm{min}$ for the reaction conditions selected in this study. The results of the simulation confirm that the benefit of the shrinking bed operation also applies to the cellulose hydrolysis. Figure 3 shows the profile of remaining cellulose (the extent of the bed shrinkage) across the reactor position. The extent of bed shrinkage reached as high as $92 \%$. Since the solid amount in the reactor is reduced, the liquid throughput can be reduced also to retain same level of concentration and yield. In this situation, we project that the acid solution can be reduced by about $50 \%$ in comparison to the non-shrinking bed operation.

\section{Effect of Temperature}


The temperatures and acid concentration are two main factors controlling the hydrolysis reactions especially in terms of substrate conversion and sugar decomposition. Reaction temperature applied for hydrolysis of biomass has been in the range of $160-210^{\circ} \mathrm{C}$ for the hemicellulose and $210-250^{\circ} \mathrm{C}$ for cellulose. With the countercurrent shrinking bed reactor, the exposure time of sugar to high temperature is reduced thus limiting its decomposition. This in turn allows the reaction to be conducted at high temperatures. The sulfuric acid concentration used in the simulation is the same as that applied in recent NREL investigation, i.e., $0.08 \mathrm{wt} \%$. It is much lower than those used in studies prior to recent NREL work. The simulation results shown in the Figure 4 indicate that the sugar yields for hemicellulose can reach $99 \%$ when the temperature is below $190^{\circ} \mathrm{C}$, and decrease slightly when the temperature is above $200^{\circ} \mathrm{C}$. The overall yield declines about $6 \%$ as the temperature is raised from $160^{\circ} \mathrm{C}$ to $210^{\circ} \mathrm{C}$. Obviously the yield of hemicellulose sugars is rather insensitive to the temperature at the acid concentration of $0.08 \mathrm{wt} \%$. The reactor processing capacity and the product concentration, however, are very sensitive to the change of temperature. The simulation results also indicate that the oligomers are the predominant product at all temperatures, however, monomeric xylose concentration increases at a faster rate than that of the oligomeric concentration as the temperature is raised from $160^{\circ} \mathrm{C}$ to $210^{\circ} \mathrm{C}$.

The effect of temperature on cellulose hydrolysis is summarized in Figure 5. Unlike hemicellulose, yield of glucose is strongly affected by temperature. The glucose yield is seen to decrease from $96 \%$ to $68 \%$ when the temperature is raised by $40^{\circ} \mathrm{C}$ from $210^{\circ} \mathrm{C}$. Similar to the hemicellulose hydrolysis, the reactor processing capacity and glucose concentration sharply increase with temperature. It may be noted that although the yield decreases sharply at high temperatures for a given liquid velocity, it is possible to improve the yield even at high 
temperature by increasing the liquid flow rate. This effect of liquid velocity is shown later in the text.

\section{Effect of Acid Concentration}

The effect of acid concentration on the hydrolysis of hemicellulose and is summarized in Figure 6 respectively. As seen in Figure 6, the acid concentration has similar effect as that of the temperature on the hemicellulose hydrolysis. That is, the maximum reactor processing capacity can be increased with slight reduction in yield as the concentration of acid is increased from 0.06 to $0.30 \mathrm{wt} \%$. Again it should be emphasized here that the slightly lower yield obtained at higher acid concentration at a given flow rate can be further improved, provided a lower concentration than that shown in the figure 6 is acceptable. This process of increasing the yield at the expense of product concentration is shown later in the text. Both xylose concentration and the reactor processing capacity increase linearly with the acid concentrations. The oligomer concentration in liquid stream increases slightly initially and then levels off to a near constant value of 2.4 $\mathrm{g} / 100 \mathrm{ml}$.

We were unable to study the effect of acid concentration on cellulose hydrolysis due to the limitation of our kinetic parameter shown in table 1 . The kinetic parameter for cellulose hydrolysis was estimated using only one acid concentration.

Although the effect of acid concentration is similar to the effect of temperature (increased reactor processing capacity and thereby increased sugar concentration), we recommend the use of temperature as the process variable for process adjustments. It is desirable to keep the acid concentration low primarily because one need not worry about recovering it and also low acid levels would generate less waste sludge and reduced disposal problems. 


\section{Effect of Acid Flow Rate}

The effects of liquid flow rate on the acid hydrolysis of hemicellulose were studied with $0.08 \mathrm{wt} \%$ sulfuric acid. The simulations were conducted under the selected temperatures at $170^{\circ} \mathrm{C}, 190^{\circ} \mathrm{C}$, and $210^{\circ} \mathrm{C}$ and its consequent $\mathrm{V}_{\mathrm{o}}$, opt. The $\mathrm{V}_{\mathrm{o}}$, opt is determined as shown in Figure 2 for the given temperature and acid concentration. As the liquid flow rate increases, one expects (1) an increase in sugar yield, since the degradation of sugars is reduced as the sugars experience a shorter residence time inside the reactor and (2) a decrease in sugar concentration because of more liquid input. Figure 7 shows that the liquid linear velocity has no effect on the yield at the relatively low temperature such as $170^{\circ} \mathrm{C}$. This is because the yield has already reached its maximum (close to $100 \%$ of theoretical). The sugar concentration (xylose+oligomer) at this temperature decreases with the liquid flow rate as expected. As the reaction temperature increases, the liquid flow rate becomes a critical factor governing the sugar yield and sugar concentration. For example, at the temperature of $210^{\circ} \mathrm{C}$, the reactor maximum processing capacity was found to be $4.29 \mathrm{~cm} / \mathrm{min}$. The sugar yield is increased substantially from 70 to $95 \%$ as the liquid flow rate is raised from $1.0 \mathrm{~cm} / \mathrm{min}$ to $2.5 \mathrm{~cm} / \mathrm{min}$. For liquid flow rates beyond 2.5 $\mathrm{cm} / \mathrm{min}$, the increase in sugar yield is marginal, but the sugar concentration continuously decreases. From these simulation results, $w$ conclude that the hydrolysis of hemicellulose at $190^{\circ} \mathrm{C}$ and with $0.08 \mathrm{wt} \%$ sulfuric acid and liquid linear velocity of $2.0 \mathrm{~cm} / \mathrm{min}$ is one set of the practical conditions for yellow poplar in terms of yield (98.5\%) and sugar concentration (2.6 $\mathrm{g} / 100 \mathrm{~mL})$.

The effects of liquid flow velocity on the cellulose hydrolysis are summarized in Figure

8. At the temperature of $210^{\circ} \mathrm{C}$, although the glucose yield is quite high, the sugar stream is 
simply too dilute. At $230^{\circ} \mathrm{C}$, the glucose yield increases from $70 \%$ to $95 \%$ as the liquid flow velocity is increased from $1.0 \mathrm{~cm} / \mathrm{min}$ to $7.0 \mathrm{~cm} / \mathrm{min}$. Further increase of the liquid flow velocity did not improve the yield but brought about only the dilution of the sugar product. At $250^{\circ} \mathrm{C}$, the glucose yield jumps abruptly from $39.5 \%$ to $85 \%$ as the liquid flow velocity is increased from $1.0 \mathrm{~cm} / \mathrm{min}$ to $7.0 \mathrm{~cm} / \mathrm{min}$. However, the glucose concentration decreased from $25.1 \mathrm{~g} / 100 \mathrm{~mL}$ to $7.6 \mathrm{~g} / 100 \mathrm{~mL}$. From these simulation results, we conclude that the hydrolysis of cellulose at $230^{\circ} \mathrm{C}$ and with $0.08 \mathrm{wt} \%$ sulfuric acid and liquid linear velocity of $5.0 \mathrm{~cm} / \mathrm{min}$ is one set of the practical conditions for yellow poplar in terms of yield (93\%) and sugar concentration $(2.4 \mathrm{~g} / 100 \mathrm{~mL})$. The acid flow velocity did not influence the conversion levels in both hemicellulose and cellulose hydrolysis.

\section{Effect of Peclet number}

Peclet number is one measure of degree of backmixing in the liquid flow. It is inversely proportional to diffusivity. A higher peclet number indicates lesser backmixing and vice-versa. The effect of peclet number on the acid hydrolysis of hemicellulose (and cellulose) were studied with $0.08 \mathrm{wt} \%$ sulfuric acid, temperature of $190^{\circ} \mathrm{C}\left(230^{\circ} \mathrm{C}\right.$ for cellulose $)$ and $\mathrm{Vo}$,opt of 1.09 $\mathrm{cm} / \mathrm{min}(0.6 \mathrm{~cm} / \mathrm{min}$ for cellulose). Figures 9 and 10 show the simulation results obtained at varying acid flow velocity at different dispersion levels. As the peclet number decreases, one expects a decrease in sugar yield, since the degradation of sugars is increased as the sugars experience a longer residence time inside the reactor due to back mixing. Figures 9 and 10 show that at any given liquid velocity, as the peclet number decreases, sugar yield also decreases. The drop in yield is more pronounced below peclet number of 3 . At peclet number $=1$, yield obtained was only $53 \%$ ( $41 \%$ for cellulose) at a flow velocity of $2 \mathrm{~cm} / \mathrm{min}$ and any increases in acid flow 
velocity brought about only marginal improvement in yield with steady dilution of the product stream. At the same point, yield obtained when peclet number $=10000$ was $99 \%(84 \%$ for cellulose). The conversion levels at at all peclet numbers were essentially the same indicating that the drop in yield was purely due to degradation of sugars due to back mixing. At peclet no $=$ 20 (obtained at NREL reactors), the model predicts that the yield for both hemicellulose and cellulose would be only marginally lower than that of the ideal countercurrent reactor $(\mathrm{Pe}=$ 10000). Furthermore, at acid velocity beyond $2.0 \mathrm{~cm} / \mathrm{min}$, the difference in yield would be practically negligible.

\section{Effect of Bed Shrinking}

The advantage of the shrinking-bed operation over the non-shrinking one is brought out well in Figure 11 for both hemicellulose and cellulose hydrolysis. The thin solid lines shown connect the yield and concentration obtained at different liquid velocity under shrinking and nonshrinking bed operation. As shown in Figure 11, at the liquid velocity of $3 \mathrm{~cm} / \mathrm{min}$, the yield for cellulose hydrolysis reached as high as $\mathbf{8 8 \%}$ under shrinking bed operation while the yield was only $69 \%$ under the non-shrinking bed operation. Also, glucose concentration obtained was much lower in the non-shrinking mode than that in the shrinking mode for the identical operating conditions. It may be noted however, that the drop in yield and concentration for cellulose hydrolysis is not entirely due to sugar degradation under non-shrinking mode. The extent of conversion was $99.9 \%$ under shrinking while it was only $81 \%$ under non-shrinking under identical process conditions. In other words, the optimum solid feeding rate was much lower under non-shrinking than that under shrinking for the same process conditions. At the liquid velocity of $3 \mathrm{~cm} / \mathrm{min}$, the maximum solid feeding rate was found to be only $0.17 \mathrm{~cm} / \mathrm{min}$ under 
non-shrinking while it was as high as $0.6 \mathrm{~cm} / \mathrm{min}$ for shrinking. Even under the reduced solid feeding rate, the yield obtained under non-shrinking is lower than that obtained under shrinking at any given level of sugar concentration. Alternatively, taking a sample point from the simulation results in order to achieve the same cellulose yield of $92 \%$ for the given conditions except the solid feeding rate, the glucose concentration is $2.4 \mathrm{~g} / 100 \mathrm{ml}$ for the bed-shrinking operation and $1.1 \mathrm{~g} / 100 \mathrm{ml}$ for non-shrinking one, $118 \%$ increase. Figure 11 also shows that the benefit of bed shrinking applies for the hemicellulose hydrolysis as well. But, the extent of bed shrinkage is much smaller for hemicellulose (35\%) than that for cellulose $(92 \%)$. Consequently, the effect of it is also much smaller for hemicellulose than it is for cellulose. 


\section{Notations}

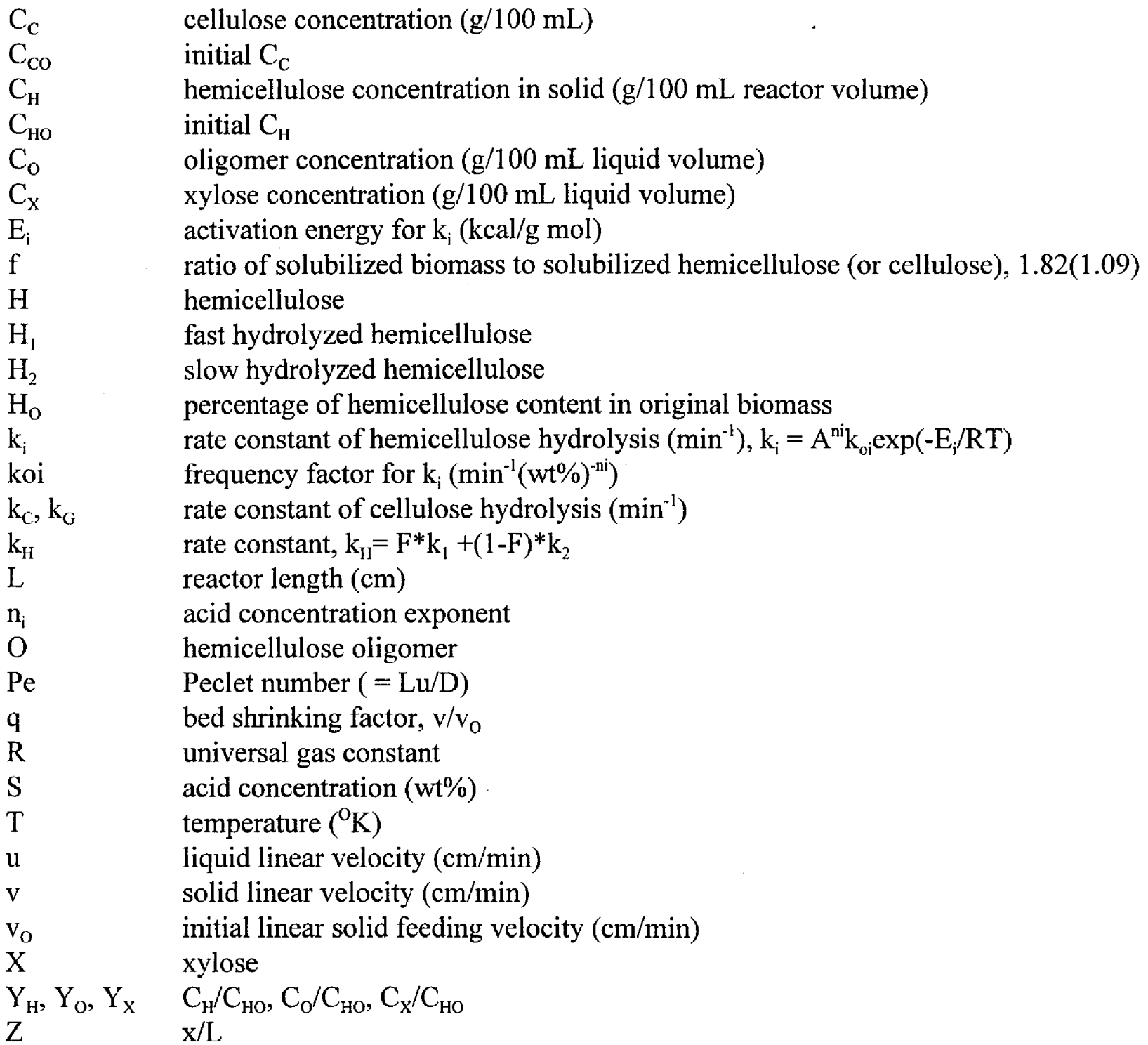

\section{Greek}

$\begin{array}{ll}\alpha_{3}, \alpha_{4} & \mathrm{k}_{3} / \mathrm{k}_{\mathrm{H}}, \mathrm{k}_{4} / \mathrm{k}_{\mathrm{H}} \\ \beta & \mathrm{k}_{\mathrm{H}} \mathrm{L} / \mathrm{u} \\ \varepsilon & \text { void fraction in bed } \\ \lambda & \rho /\left(\mathrm{fH}_{\mathrm{O}}\right) \\ \rho & \text { bulk density } \\ \omega & \mathrm{u} / \mathrm{v}_{\mathrm{O}}\end{array}$


Table 1. Kinetic parameters from previous investigations

\begin{tabular}{|c|c|c|c|c|}
\hline \multirow[b]{2}{*}{$\begin{array}{l}\text { Hemicellulose }^{a} \\
\text { (Chen et al, 1996) }\end{array}$} & \multicolumn{4}{|c|}{$\begin{array}{l}\mathrm{k}_{\mathrm{i}}=\mathrm{k}_{\mathrm{io}}\left(\mathrm{C}_{\mathrm{A}}\right)^{\mathrm{ni}} \exp \left(-\mathrm{E}_{\mathrm{i}} / \mathrm{RT}\right) \text { for hemicellulose } \\
\mathrm{k}_{\mathrm{i}}=\mathrm{k}_{\mathrm{io}} \exp \left(-\mathrm{E}_{\mathrm{i}} / \mathrm{RT}\right) \text { for cellulose }\end{array}$} \\
\hline & $\mathrm{k}_{\mathrm{i}}$ & $\begin{array}{c}\mathrm{k}_{\mathrm{io}} \\
\min ^{-1}(\mathrm{wt} \%)^{-\mathrm{ni}}\end{array}$ & $\mathrm{n}_{\mathrm{i}}$ & $\begin{array}{c}\mathrm{E}_{\mathrm{i}} \\
\mathrm{kcal} / \mathrm{g} \text { mol }\end{array}$ \\
\hline & 1 & $1.458 \times 10^{15}$ & 1.0 & 30.9 \\
\hline & 2 & $1.300 \times 10^{14}$ & 0.5 & 30.0 \\
\hline & 3 & $6.372 \times 10^{10}$ & 1.5 & 21.0 \\
\hline & 4 & $1.618 \times 10^{12}$ & 0.8 & 27.5 \\
\hline \multirow[t]{3}{*}{$\begin{array}{l}\text { Cellulose }^{\mathrm{b}} \\
\text { (Wu et al, 1997) }\end{array}$} & $\mathrm{k}_{\mathrm{i}}$ & $\begin{array}{c}\mathrm{k}_{\mathrm{i}} \\
\min ^{-1}(\mathrm{wt} \%)\end{array}$ & & $\begin{array}{c}\mathrm{E}_{\mathrm{i}} \\
\mathrm{kcal} / \mathrm{g} \mathrm{mol}\end{array}$ \\
\hline & 1 & $1.4 \times 10^{18}$ & & 44.511 \\
\hline & 2 & $1.81 \times 10^{13}$ & & 33.215 \\
\hline
\end{tabular}

\footnotetext{
${ }^{a}$ Kinetic study conducted using Yellow Poplar Sawdust as the substrate at the temperature of $160-190^{\circ} \mathrm{C}$ and $0.049-0.196 \mathrm{wt} \%$ sulfuric acid.

${ }^{\mathrm{b}}$ Kinetic study conducted using Yellow Poplar Sawdust as the substrate at temperature of 205$235^{\circ} \mathrm{C}$ and $0.064 \mathrm{wt} \%$ sulfuric acid.
} 


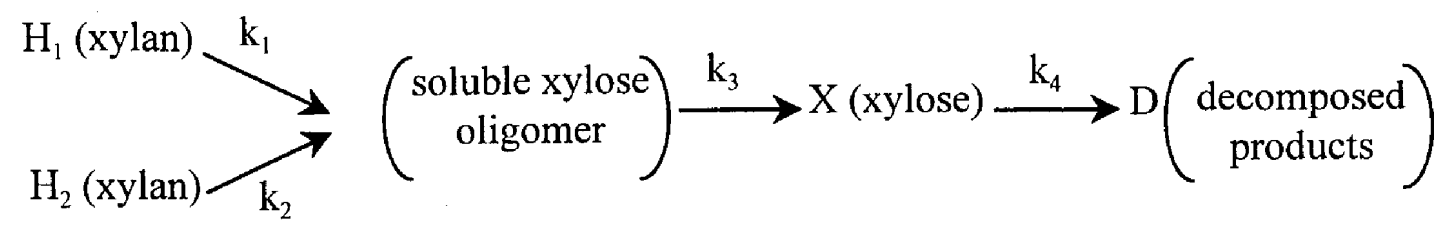

(A) Kinetic pattern of hemicellulose hydrolysis. $\mathrm{H}_{1}$ refers to the easily hydrolyzed hemicellulose fraction, and $\mathrm{H}_{2}$ refers to the difficult hydrolyzed portion.

Cellulose $\stackrel{k_{c}}{\longrightarrow}$ Glucose $\stackrel{k_{i}}{\longrightarrow}$ Decomposed products

(B) Kinetic pattern of cellulose hydrolysis

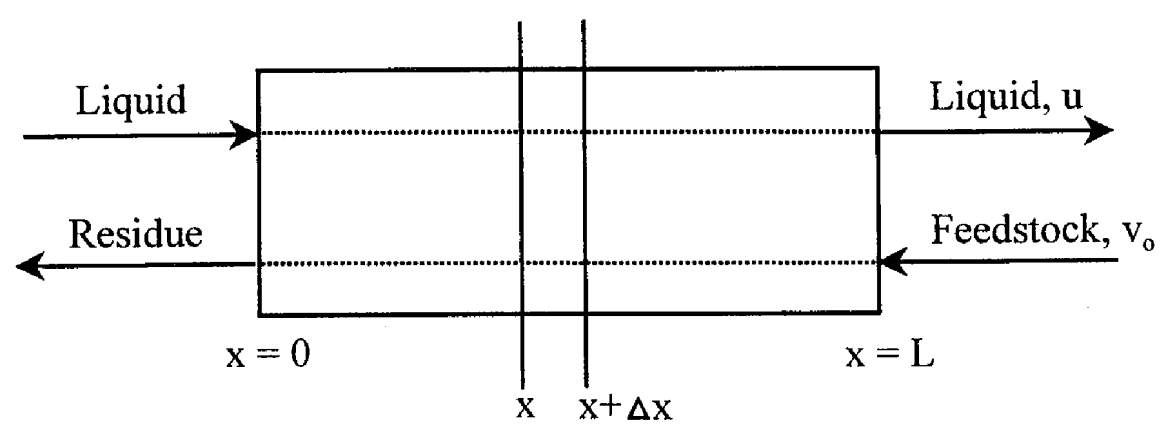

(C) Countercurrent reactor

Figure 1. Kinetic pathway of acid hydrolysis of lignocellulosic biomass and conceptual sketch of countercurrent reactor. 

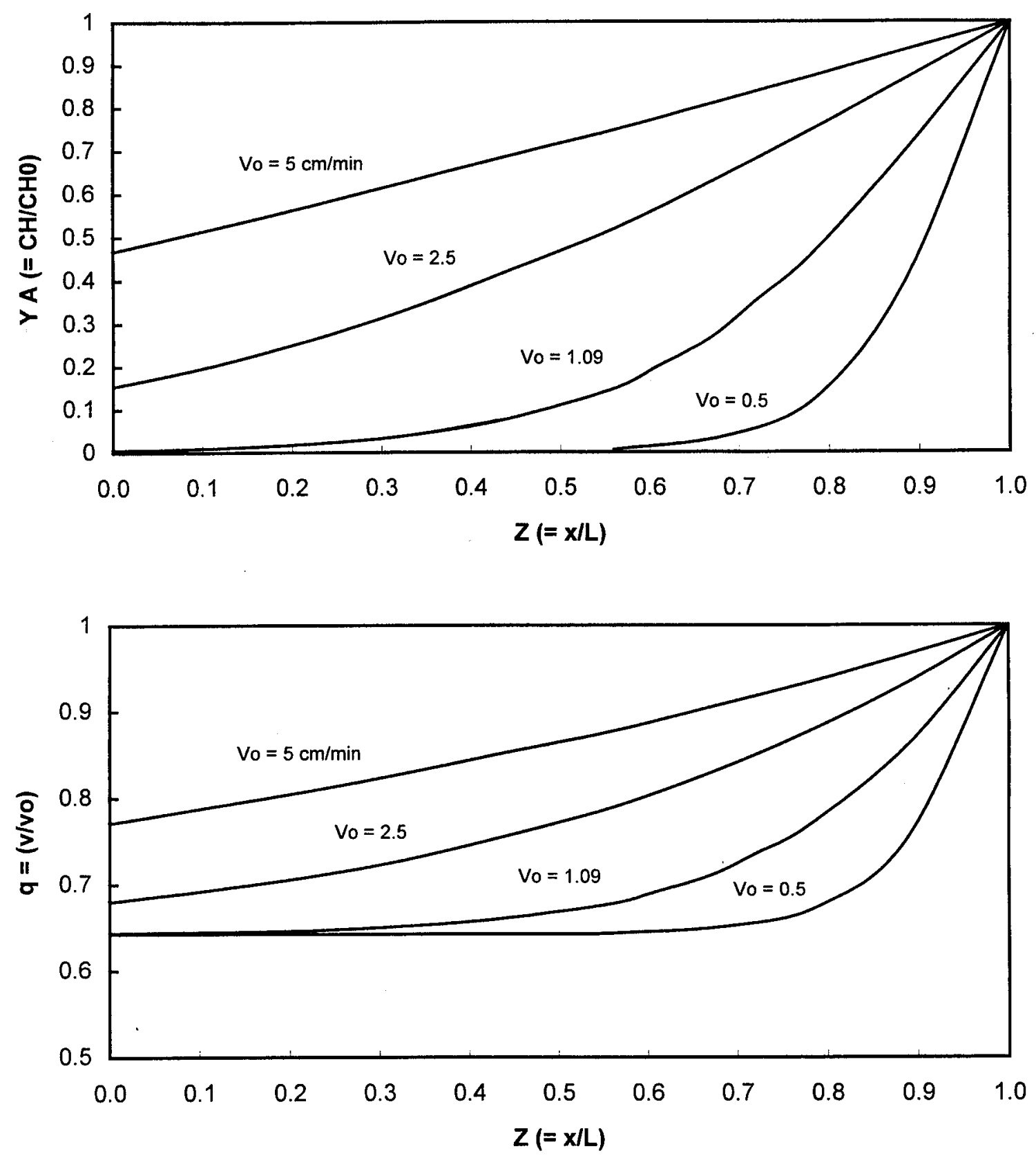

Figure 2. Effect of initial solid feeding rate on the hydrolysis of hemicellulose. Assumption: $T=$ $190 \mathrm{C}, u=2 \mathrm{~cm} / \mathrm{min}$, acid concentration $=0.08 \mathrm{wt} \%$ sulfuric acid, peclet no $=20$. 

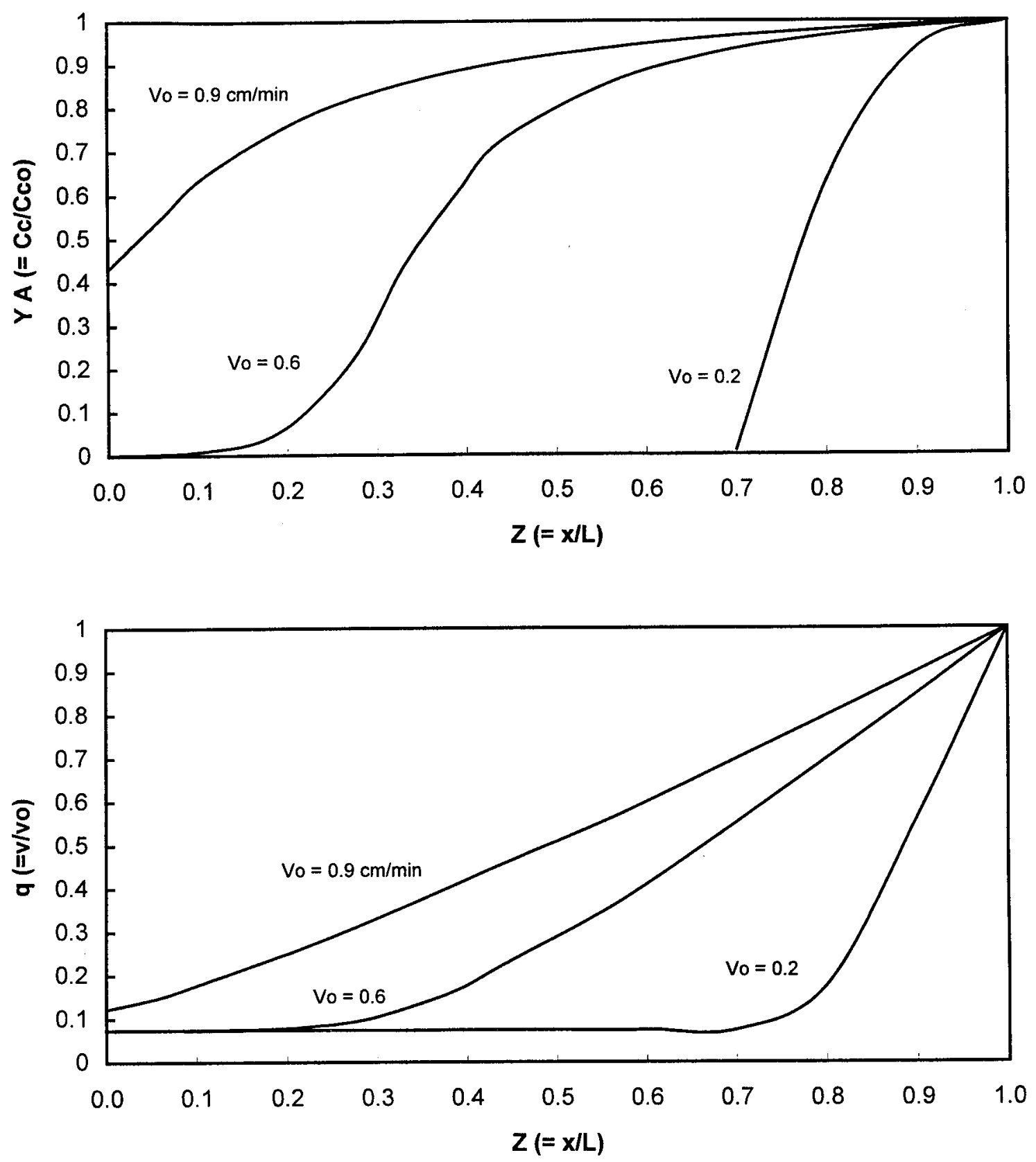

Figure 3. Effect of initial solid feeding rate on the hydrolysis of cellulose. Assumption: $T=$ $230 \mathrm{C}, \mathrm{u}=3 \mathrm{~cm} / \mathrm{min}$, acid concentration $=0.08 \mathrm{wt} \%$ sulfuric acid, peclet no $=20$. 

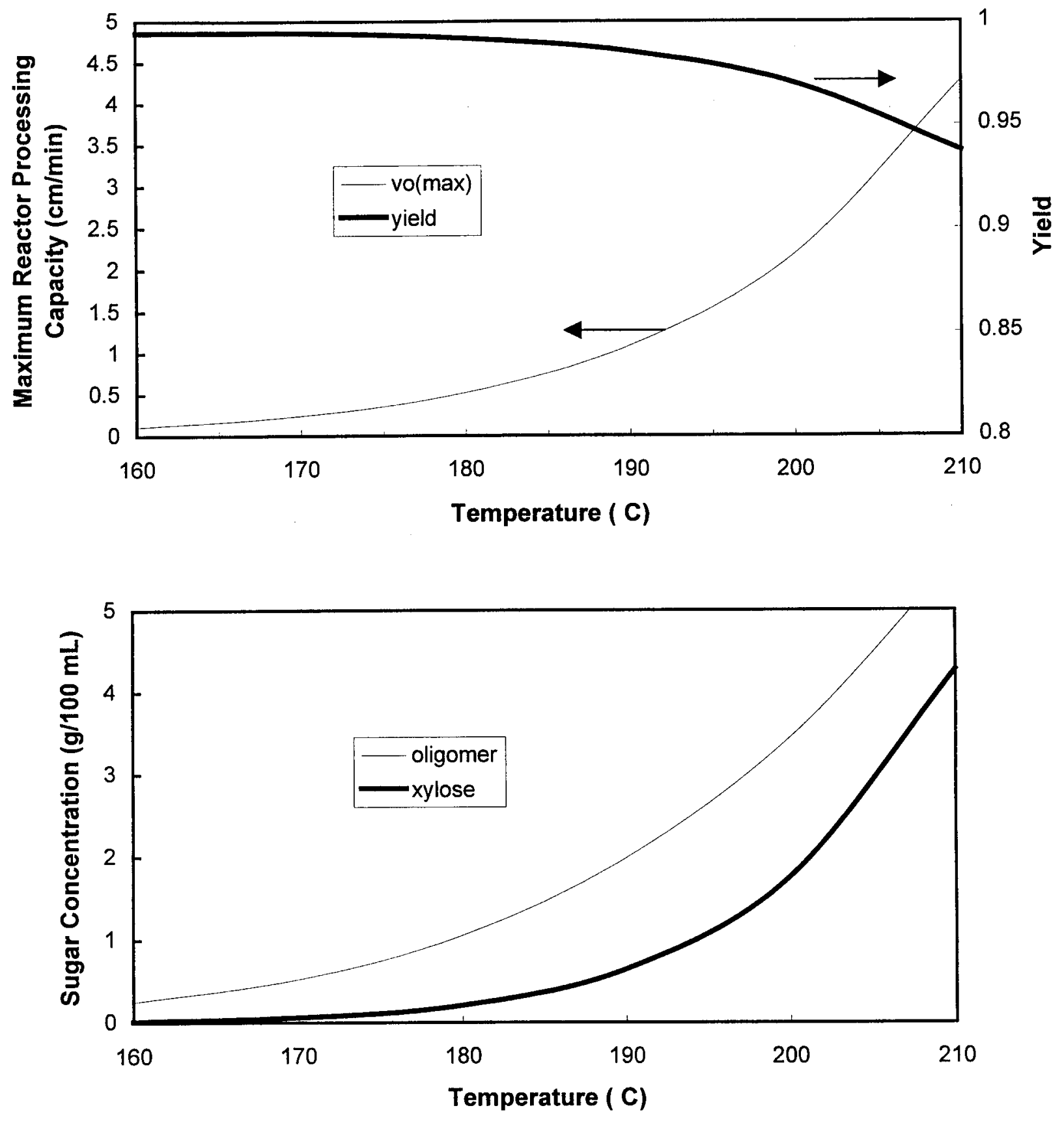

Figure 4. Effect of temperature on the hydrolysis of hemicellulose. Assumption: $u=2 \mathrm{~cm} / \mathrm{min}$ and acid concentration $=\mathbf{0 . 0 8} \mathbf{w t} \%$ sulfuric acid, Peclet no $=20$. 

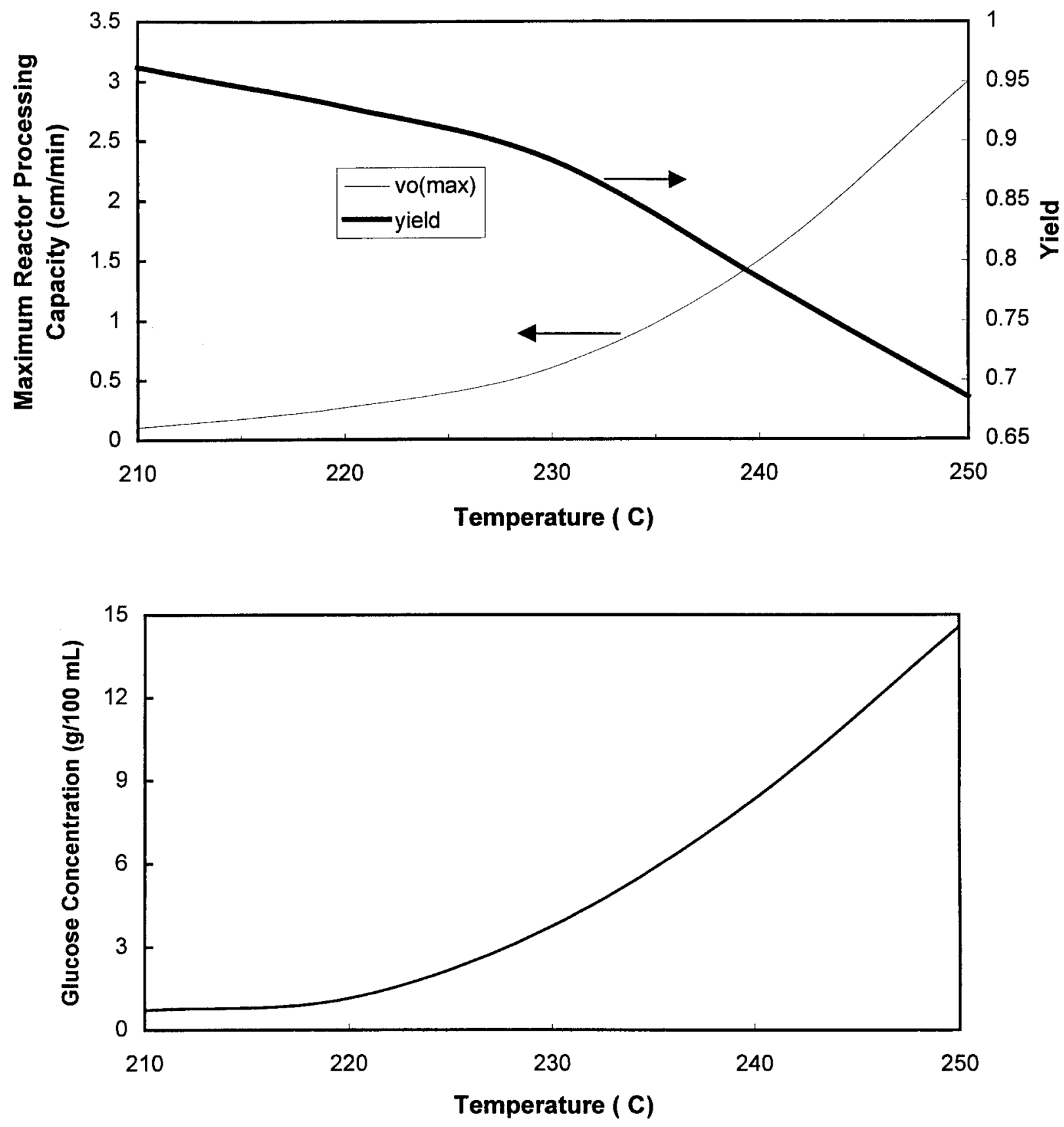

Figure 5. Effect of temperature on the hydrolysis of cellulose. Assumption: $u=3 \mathrm{~cm} / \mathrm{min}$ and acid concentration $=0.08$ wt $\%$ sulfuric acid, Peclet no $=20$. 

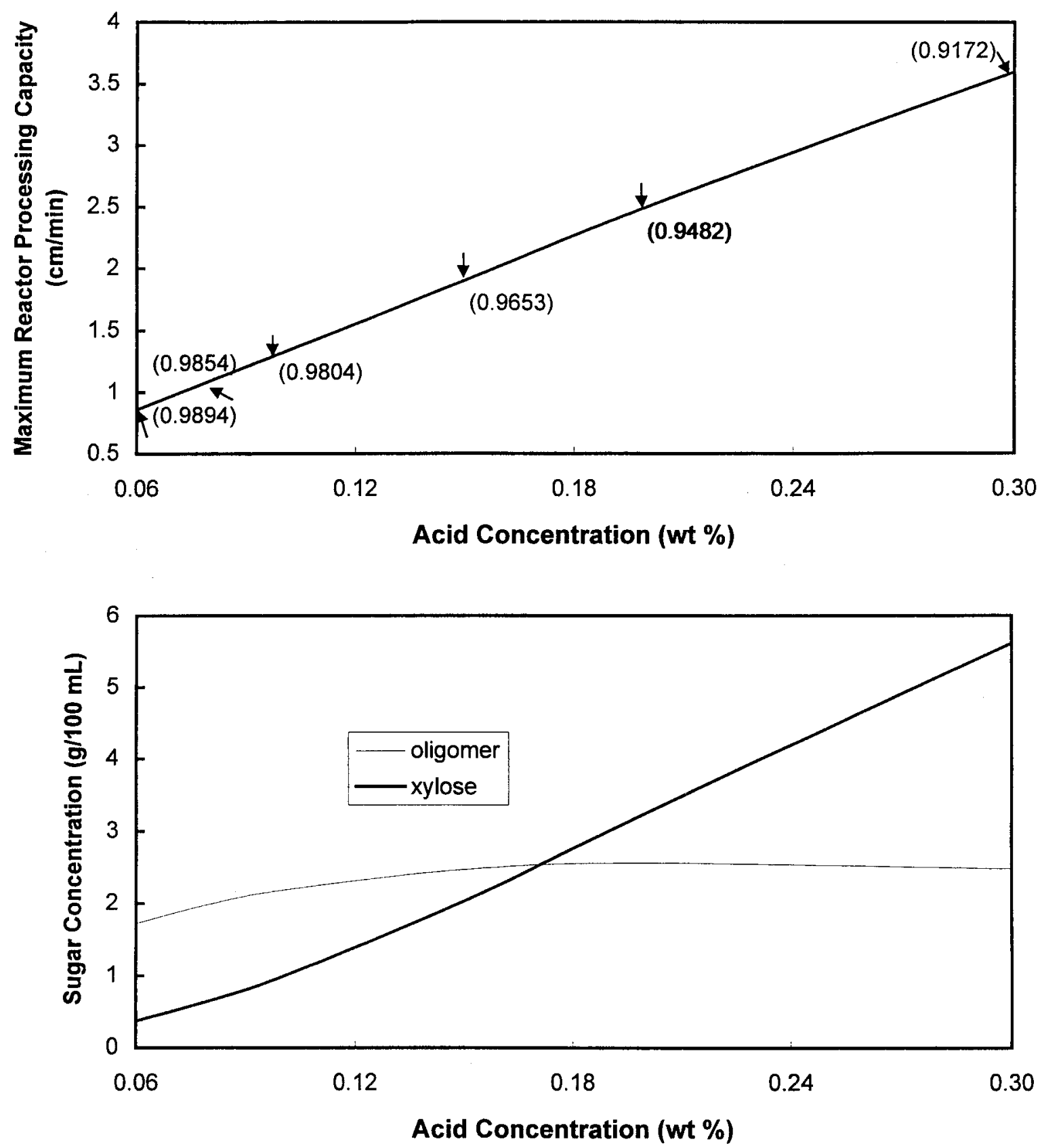

Figure 6. Effect of acid concentration on the hydrolysis of hemicellulose. Assumption: $u=2 \mathrm{~cm} / \mathrm{min}$ and $\mathrm{T}=190 \mathrm{C}$, Peclet no $=20$. 

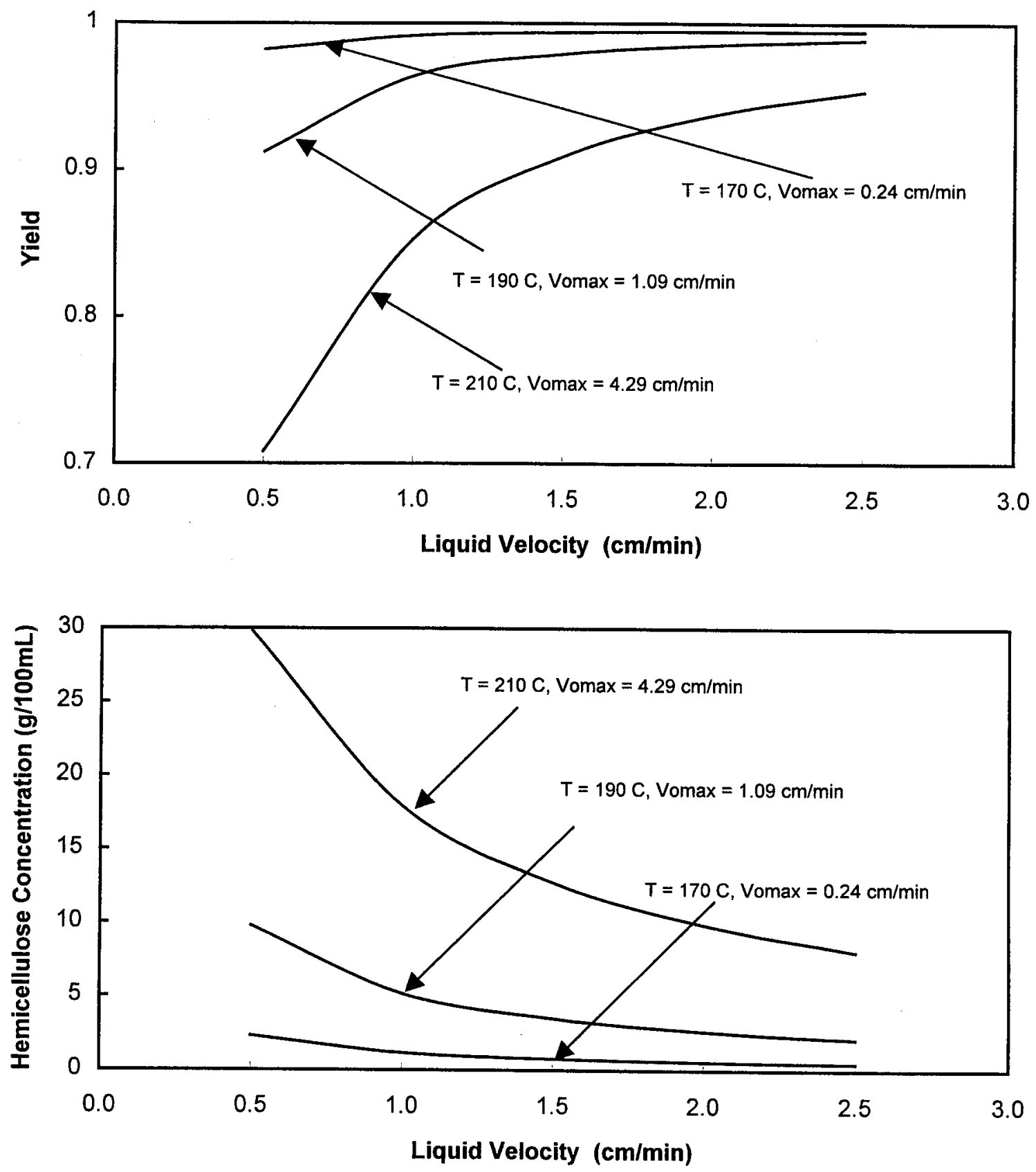

Figure 7. Effect of acid flow velocity on the hydrolysis of hemicellulose at different temperatures. Assumption: acid concentration $=0.08$ wt $\%$, Peclet no $=20$ 

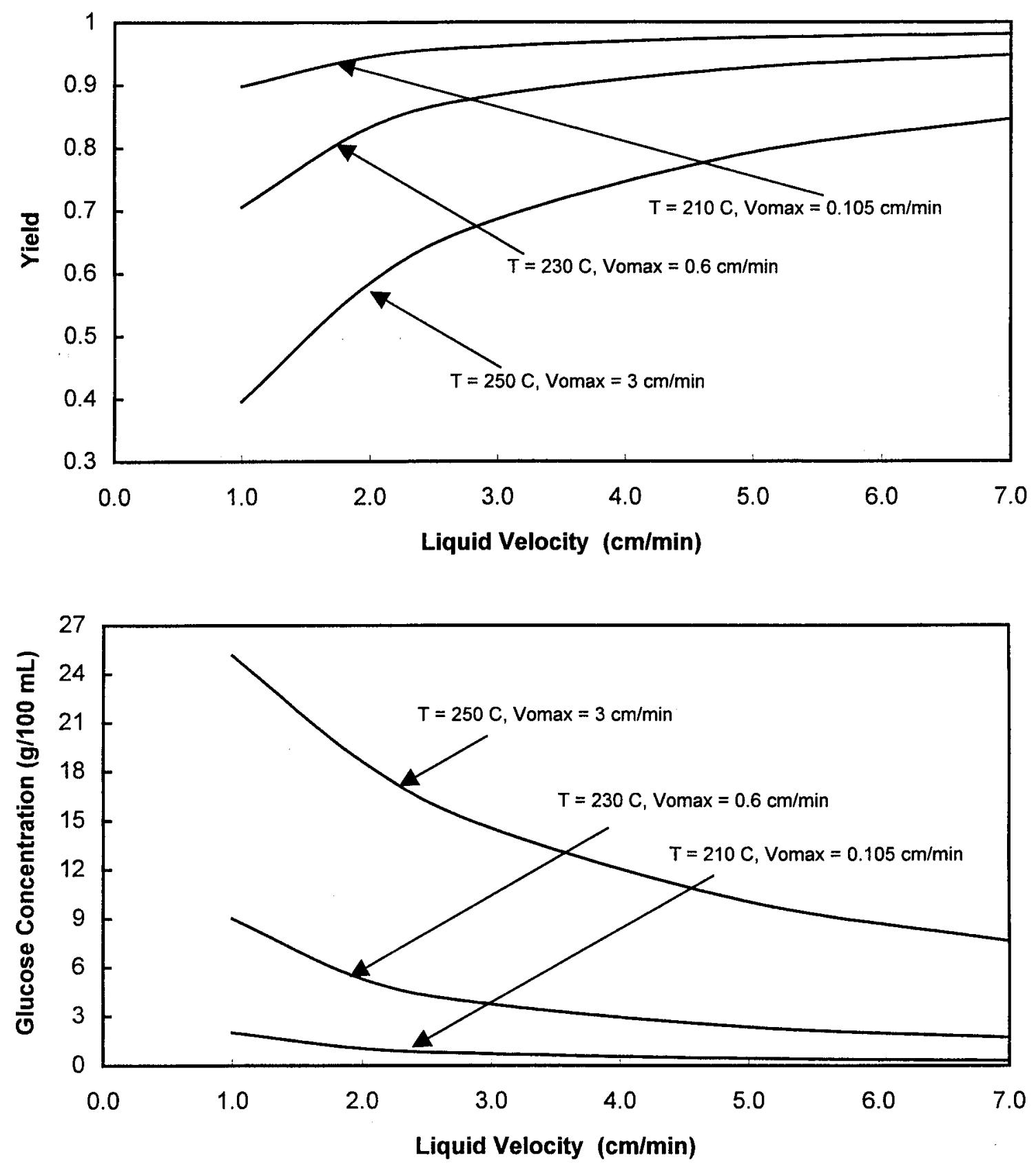

Figure 8. Effect of acid flow velocity on the hydrolysis of cellulose at different temperatures. Assumption: acid concentration $=0.08 w t \%$, Peclet no $=20$ 

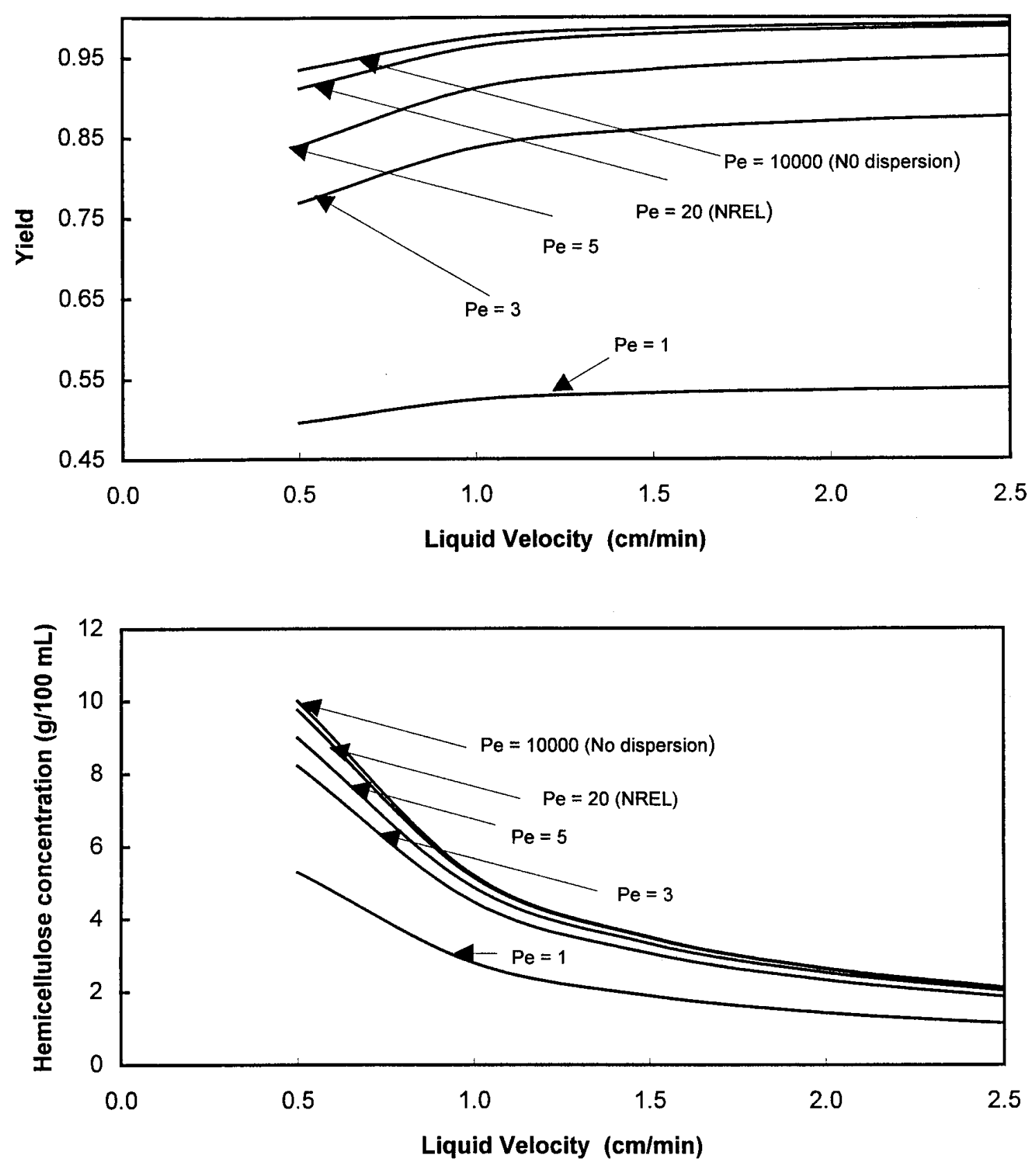

Figure 9. Effect of acid flow velocity on the hydrolysis of hemicellulose at different dispersion levels. Assumption: $\mathrm{T}=190 \mathrm{C}$, Vomax $=1.09 \mathrm{~cm} / \mathrm{min}$, acid concentration $=0.08 \mathrm{wt} \%$ 

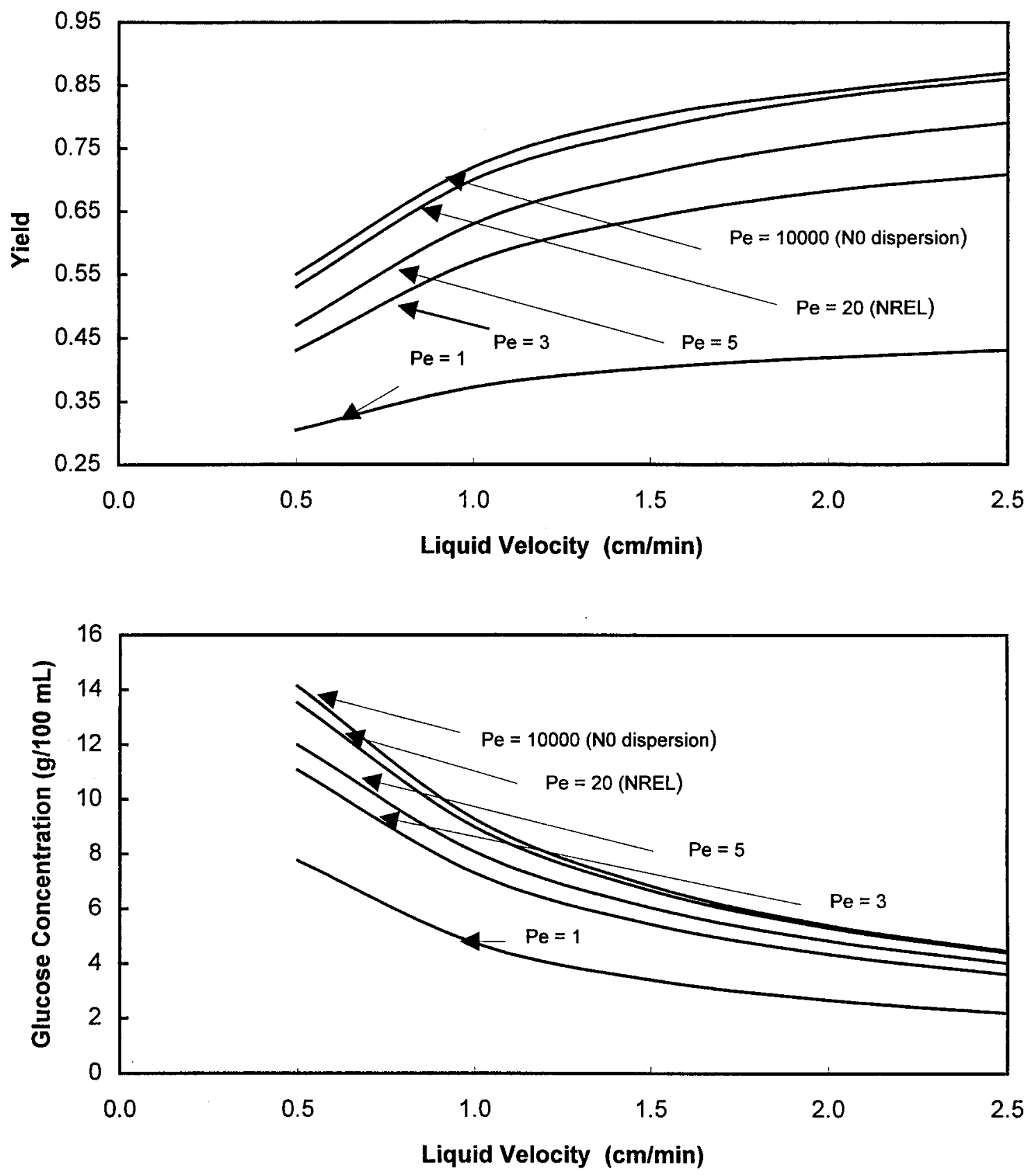

Figure 10. Effect of acid flow velocity on the hydrolysis of cellulose at different dispersion levels. Assumption: $\mathrm{T}=230 \mathrm{C}$, Vomax $=0.6 \mathrm{~cm} / \mathrm{min}$, acid concentration $=0.08 \mathrm{wt} \%$ 

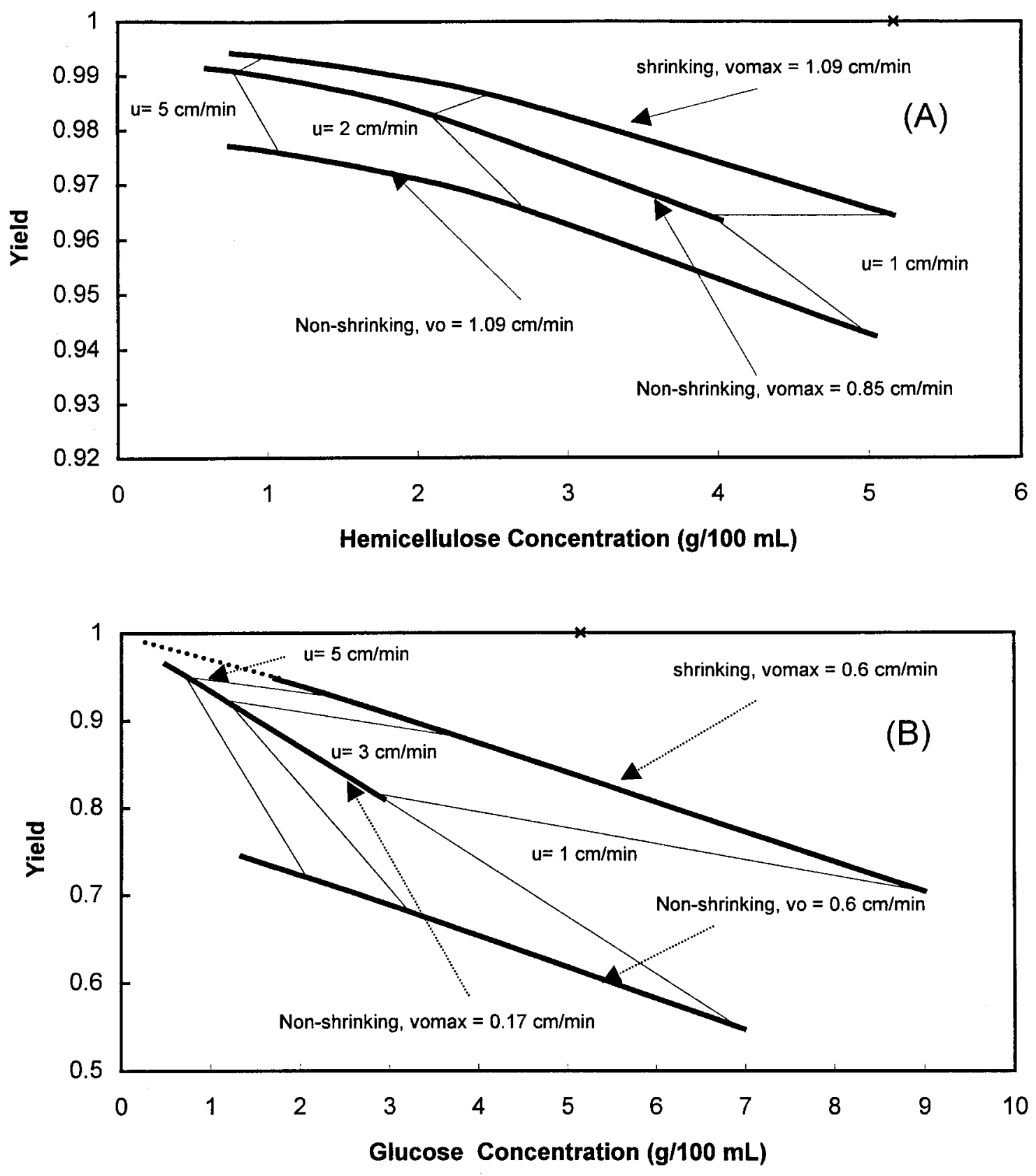

Figure 11. Yield vs. sugar product concentration under shrinking and non-shrinking operation mode.

(A) Hemicellulose hydrolysis, (B) Cellulose hydrolysis. Assumptions: acid concentration $=0.08 w t \%, P e=20, T=190 \mathrm{C}$ for hemicellulose hydrolysis and $\mathrm{T}=230 \mathrm{C}$ for cellulose hydrolysis. 


\section{Task 2: Verification of Hydrolysis Kinetics of Cellulose of Yellow Poplar Sawdust using 0.07 wt \% Suulfuric Acid}

The kinetic investigation was conducted in two separate phases; decomposition of pure glucose, hydrolysis of glucan in Yellow Poplar Sawdust. In the method, the level of glucose was measured for the former, and the remaining glucan for the latter. The glucan hydrolysis was done for conversion up to $60 \%$. The kinetics at higher conversion is left as a future task.

\section{Experiment:}

Kinetic experiments on glucan hydrolysis by acid and on glucose decomposition were conducted in a 6-inch nickel tube reactors. The reactor were sealed at both ends with Swagelok caps. The nickel tube was obtained from Salem Tube, Inc., Greenville, PA. The supplier's test report indicates that the purity of the nickel is $>99.5 \%$.

Two oil baths were used for temperature control of reactor tubes. One of the two oil baths was set at $250 \mathrm{C}$ to reduce the pre-heating time. The reactor tubes were first placed in this bath and moved to another bath set at desired reaction temperature. Preheating time ranged between 45 to 70 seconds depending on the reaction temperature chosen. Four levels of temperature were applied: $205 \mathrm{C}, 215 \mathrm{C}, 225 \mathrm{C}$ and $235 \mathrm{C}$. At the end of the reaction, the reactors was quickly chilled with ice-water to terminate the reaction.

Glucose concentration of $6 \mathrm{~g} / \mathrm{L}$ was used for all glucose decomposition experiments. For the cellulose hydrolysis experiments, dilute-acid pretreated yellow poplar was used as the substrate. Pretreatment conditions were, $7 \mathrm{mM}$ sulfuric acid, $150 \mathrm{C}, 15 \mathrm{~min}, 195 \mathrm{C}, 15 \mathrm{~min}$. The pretreated substrate contained $69.5 \mathrm{wt} \%$ glucan and $28.0 \mathrm{wt} \%$ klason lignin on a dry basis. The pretreated yellow poplar was stored in the cool room under wet condition (without water 
washing). Moisture content was determined to be $84 \%$. For all kinetic experiments, a sulfuric acid solution of $7 \mathrm{mM}(0.0684 \mathrm{wt} \%)$ was used. The reactor was packed with $0.9 \mathrm{~g}$ substrate and $9 \mathrm{~mL}$ acid solution to achieve a solid to liquid ratio of 1:10. A reaction time of less than 7 minutes was chosen for glucose decomposition experiments whereas reaction time up to 30 minutes were applied for cellulose hydrolysis. After reaction, solids remaining were collected and washed. The overall weight remaining and glucan content were determined. The glucan content remaining after the reaction was used as input data in determination kinetic parameters.

\section{Data Analysis:}

A first order reaction model was used to determine kinetic parameters. Linear regression was employed to determine the first order rate constants (Equation $\left.\ln (\mathrm{CAO} / \mathrm{Ca})=\mathrm{K}^{*} \mathrm{t}\right)$. The oligomers of glucose was not found in the hydrolyzates of cellulose. Since only one level of acid concentration was used in the kinetic study, the rate constant equation was simplified to $\mathrm{K}=\mathrm{ko} * \exp (-\mathrm{E} / \mathrm{RT})$

The experimental results are shown in Figures $1 \& 2$. From these data, $\mathrm{k}$ values at different temperature were determined by linear regression. Then using these $\mathrm{K}$ values, $\mathrm{E}$ and ko were determined from the Arrhenius plot. The activation energy(E) and the frequency factor (ko) thus determined for the respective rate constants are as follows. obtained are produced below.

Glucose decomposition:

$$
\mathrm{ko}=1.81 \mathrm{E}+13
$$

$$
E=33.215 \mathrm{kcal} / \mathrm{mol}
$$

Cellulose hydrolysis:

$$
k o=1.4 \mathrm{E}+18
$$




\section{$E=44.511 \mathrm{kcal} / \mathrm{mol}$}

It is duly noted here that the activation energy for hydrolysis is substantially higher than that of sugar decomposition even at high temperature and low acid conditions. 

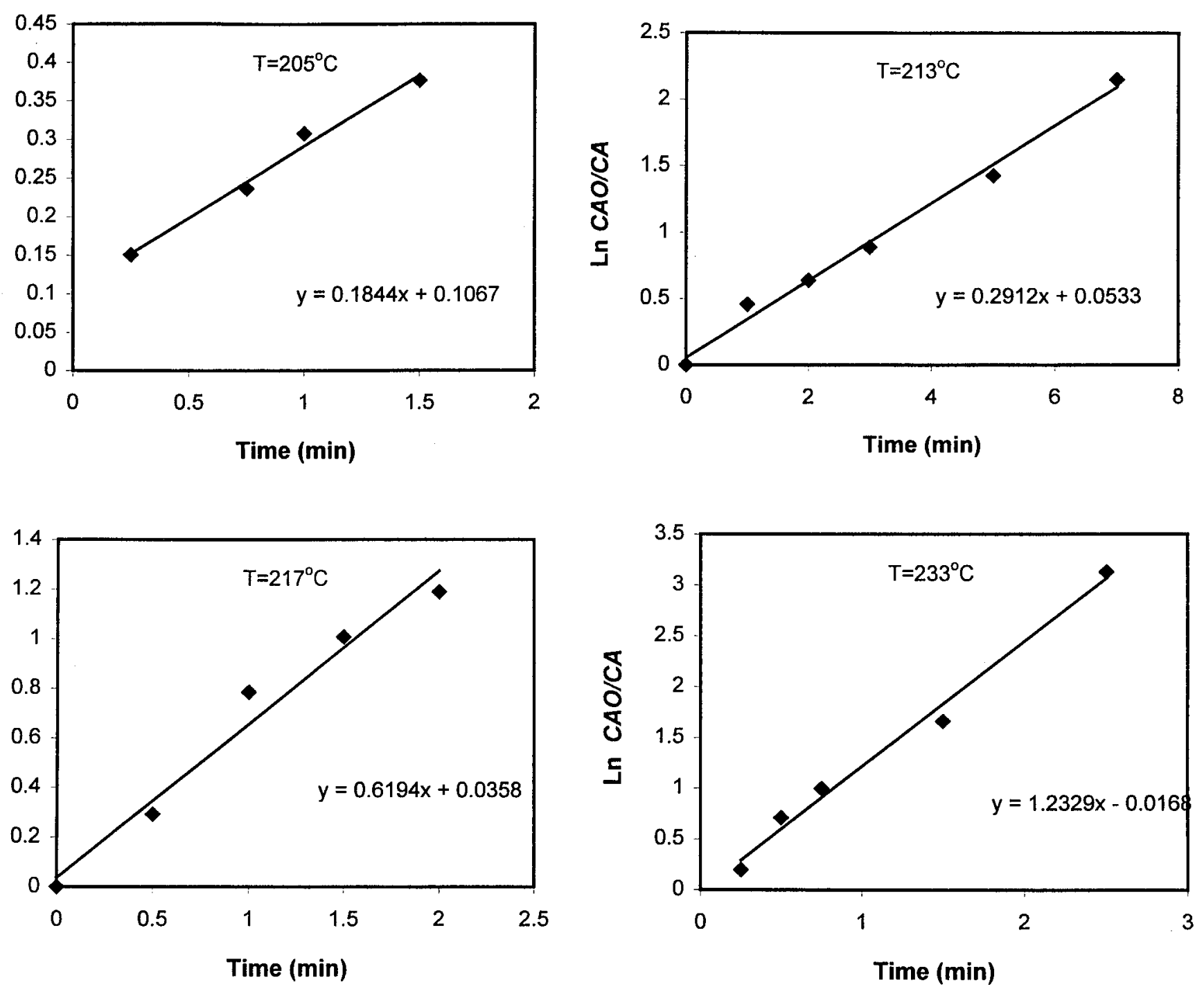

Fig. 1 Batch kinetic data for glucose decomposition, $0.0686 w t \%$ sulfuric acid 

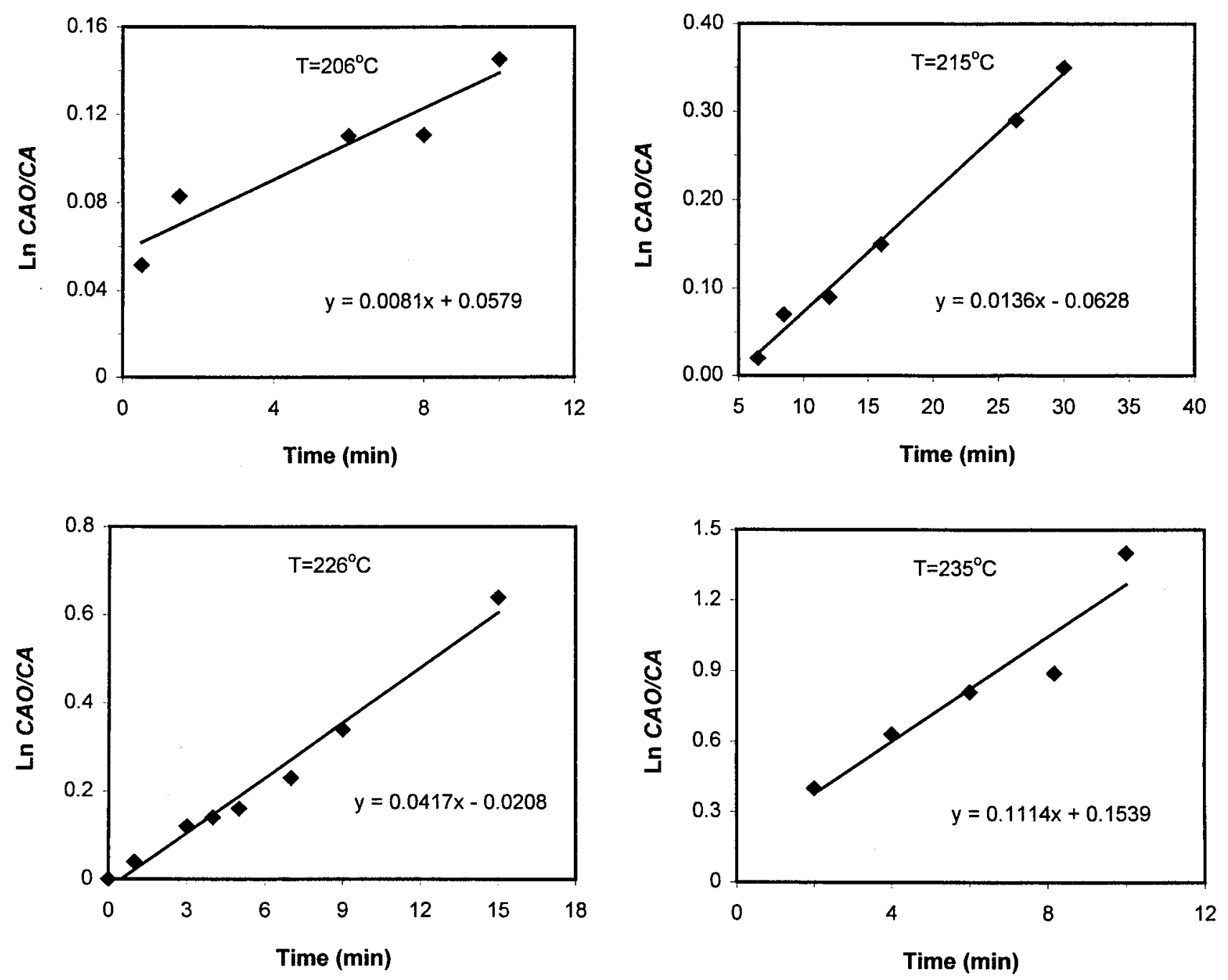

Fig.2 Batch kinetics data for cellulose hydrolysis, $0.0686 \mathrm{wt} \%$ sulfuric acid (Measured by remaining cellulose) 
Batch data set, cellulose hydrolysis, $7 \mathrm{mM}$ sulfuric acid

$\begin{array}{cccccccc}T=206.4 C & T(K) & 1 / T * 1000 & -I n k & k c & k o= & 1.4 E+18 & \\ 206.4 & 479.55 & 2.085288 & -4.815891 & 0.0081 & E= & 44.511 & \mathrm{kcal} / \mathrm{mol} \\ 215.5 & 488.65 & 2.046455 & -4.297685 & 0.0136 & & & \\ 226.4 & 499.55 & 2.001802 & -3.153556 & 0.0427 & & & \\ 235 & 508.15 & 1.967923 & -2.194628 & 0.1114 & & \end{array}$

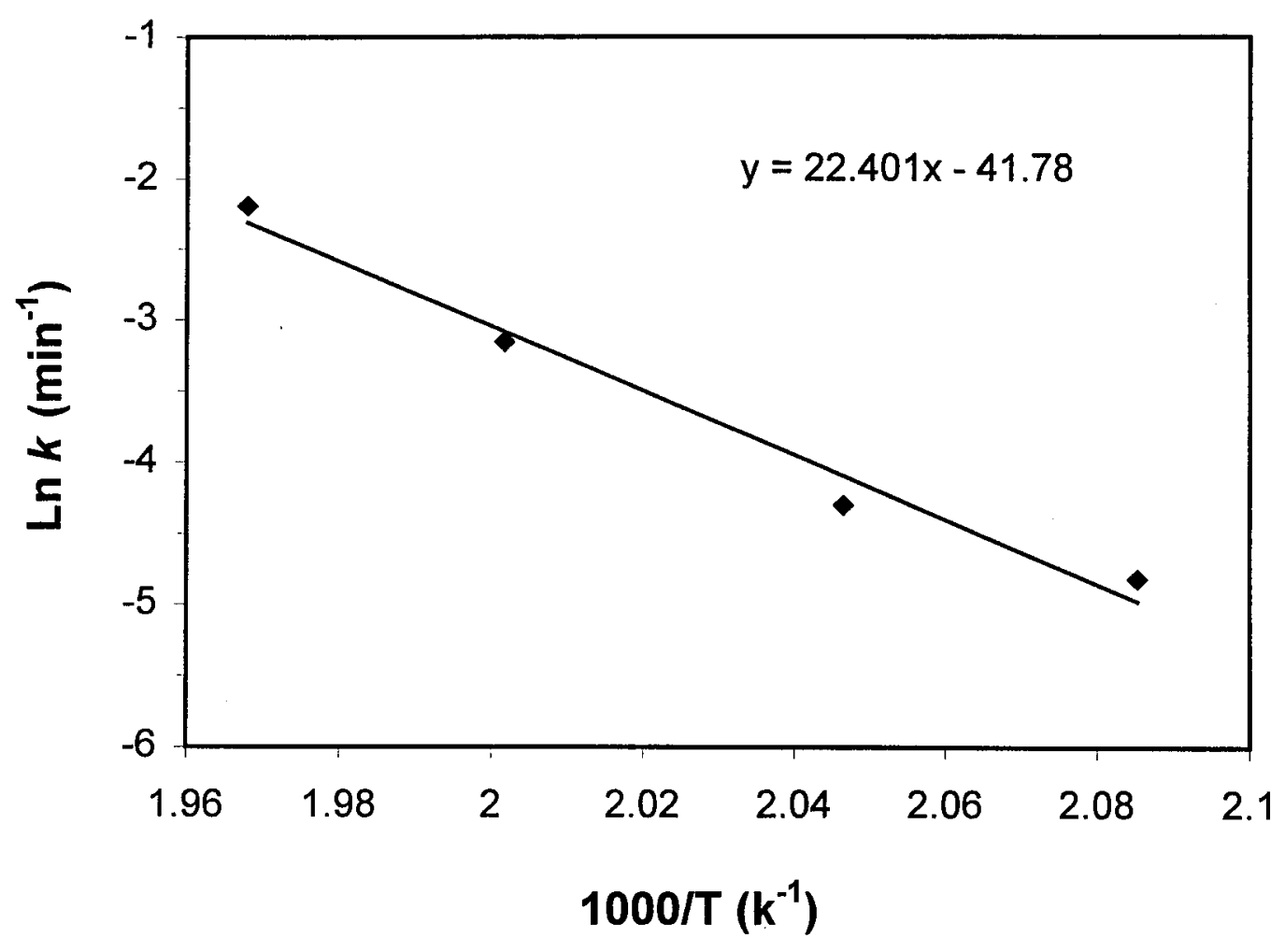

Fig. 3 Arrhenius plot for cellulose hydrolysis, $0.0686 w t \%$ sulfuric acid (Substrate: dilute-acid pretreted yellow poplar sawdust) 
Batch data set, glucose degradation

$\begin{array}{lcccclll}T(C) & T(K) & 1000 / T & \text { Ink } & k & & & \\ 205 & 478.15 & 2.091394 & -1.69119 & 0.1843 & k= & 1.81 \mathrm{E}+13 & \\ 213 & 486.15 & 2.056978 & -1.233745 & 0.2912 & \mathrm{E}= & 33.215 & \mathrm{kcal} / \mathrm{mol} \\ 217 & 490.15 & 2.040192 & -0.486296 & 0.6149 & & & \\ 233 & 506.15 & 1.975699 & 0.209369 & 1.2329 & & & \end{array}$

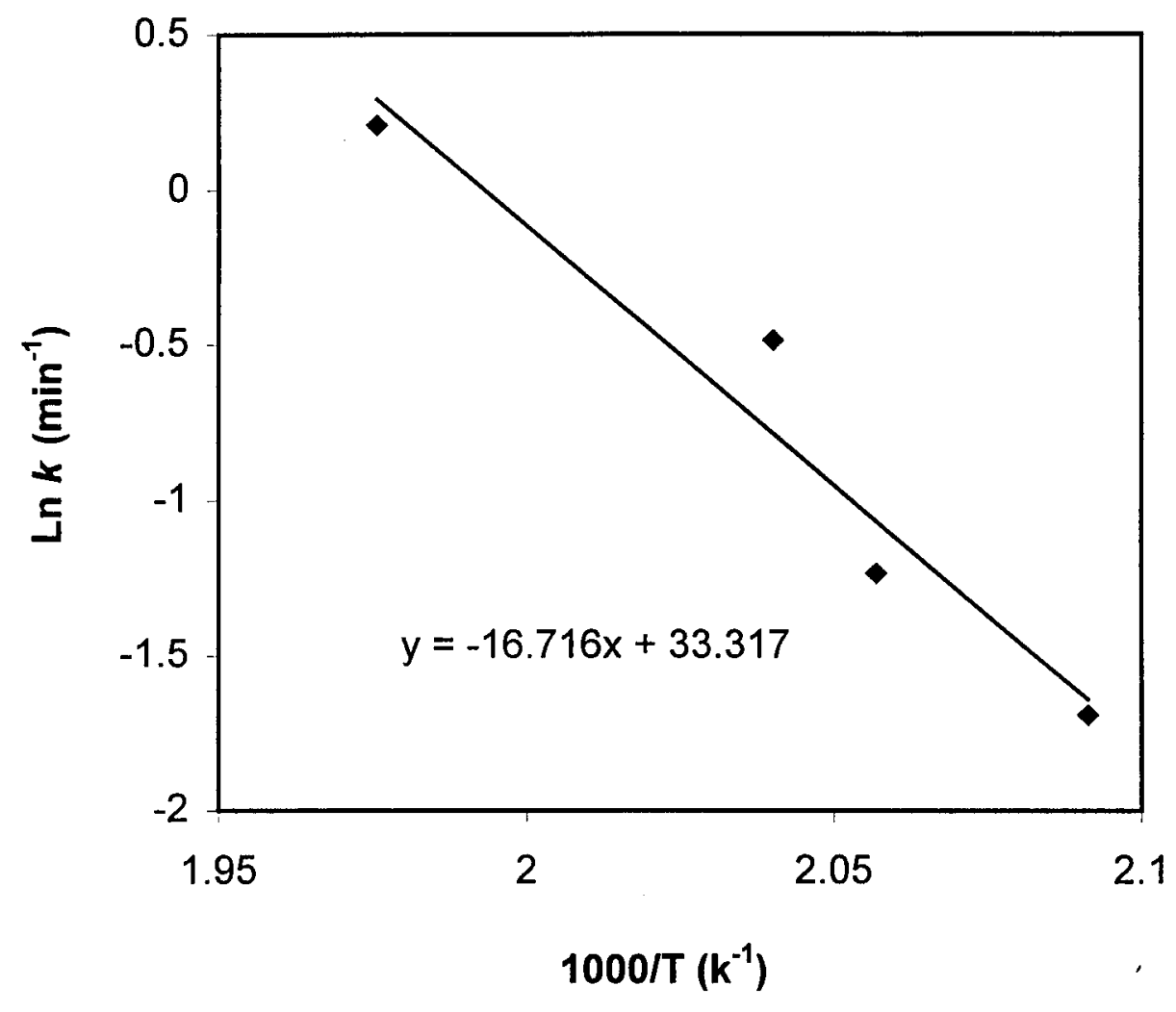

Fig.4 Arrhenius plot for glucose decomposition, $0.0686 w t \%$ sulfuric acid 


\section{Task 3: Preliminary Investigation on Thermal/Oxidative Cracking of Lignin Generated from Total Hydrolysis process}

\section{Oxidative Degradation of Lignin by Hydrogen Peroxide}

Lignins are complex amorphous phenolic polymers. Their composition in various biomass species ranges from $18 \%$ to $33 \%$ by weight. The aromatic complex nature of lignins suggests that they can be converted into low-molecular-weight compounds, potential precursors for fuels or chemicals. Various approaches have been taken for this purpose including hydrotreating and pyrolysis. Mild hydrodeoxygenation and dealkylation can give a mixture of substituted phenolic compounds and hydrocarbons as main products. The phenolics can be converted to methyl or ethyl aryl ethers (MAE or EAE). The mixture of alkyl aryl ethers and hydrocarbons may become a low-vapor-pressure octane enhancer that is fully compatible with gasoline.

Chemically, lignin is very reactive. It is easily oxidizable and can undergo a variety of acid- and based-catalyzed hydrolysis reactions. Oxidized by alkaline nitrobenzene, lignin provides a high yield of vanillin. The yield of 20 to $28 \%$ vanillin is typical for spruce lignin. Various degradation products from the permanganate oxidation of spruce lignin have been identified. Hemipinic acid and the biphenyls, diphenyl ethers, and the benzene polycarboxylic acids are among that group detected along with the main product of veratric isohemipinic, and metahemipinic acids. Hydrogen peroxide is a very strong oxidation reagent which has been successfully used in waste water treatment to remove organic material. The advantage of using hydrogen peroxide as the oxidation reagent is that there is no residuals left after the reaction and it is quite safe to handle. The oxidative degradation of lignin degradation using hydrogen peroxide is an interesting concept that deserves further investigation. There has been a substantial research work conducted on utilizing hydrogen peroxide in treatment of solid wastes such as waste carpet 
remnants and waste plastics by a research team in the Chemical Engineering Department in Auburn University. With the aid of this group, we have carried out a series of preliminary investigation on oxidative degradation of lignin by aqueous hydrogen peroxide near critical temperature of water.

\section{Experimental}

\section{Materials:}

A commercial grade Kraft lignin (brand name of Indulin) was supplied by Westvaco, Charleston, SC. Hydrogen peroxide purchased as $30 \%$ solution from Fisher was used after dilution to $10 \%$ and $5 \%$. Methylene Chloride was used as the extraction reagent for GC/MS analysis.

\section{Batch Reaction:}

Batch reactions were carried out using tubing bomb reactors (SS-316, 3/4", $11 \mathrm{~cm}^{3}$ in volume) capped at both ends with Swagelok screw caps. The reaction was carried out in a temperature controlled electric oven (Thermolyne 314). A $250 \mathrm{rpm}$ agitation was applied to the reactor assembly during the reaction for mixing of reactor content. For a given reaction temperature, the amount of liquid (water) to put into the reactor was calculated from steam table pressure data. At the completion of reaction, the reactor was quenched in tab water. The reactor content was brought into contact with methylene chloride for extraction. The resulting liquid sample samples (methylene chloride phase) were analyzed by GC/MS.

\section{Reaction Conditions:}

Four batch reactions have been carried out at various conditions. The detailed reaction conditions are shown in Table 1. Tests 1 and 2 are at supercritical condition of water. After the reaction the lignin powder (solids) have totally disappeared. The remaining liquid showed light tan color. Gaseous products were also formed from the reaction. It is quite obvious that all of the lignin has reacted, perhaps overreacted. The tests made here represent only sketchy spot checks. Further experiments would be needed to 
identify proper reaction conditions in terms of including temperature, reaction time, and reactant ratio (lignin/ $\mathrm{H}_{2} \mathrm{O}_{2}$ ).

Table 1: Reaction Conditions

\begin{tabular}{lcccc}
\hline Reaction Condition & Test 1 & Test 2 & Test 3 & Test 4 \\
\hline Temperature ${ }^{0} \mathbf{C}$ & 400 & 400 & 350 & 300 \\
Pressure (bar) & 350 & 350 & 250 & 100 \\
Reaction Time & $30 \mathrm{~min}$ & $15 \mathrm{~min}$ & $30 \mathrm{~min}$ & $30 \mathrm{~min}$ \\
Lignin (g) & 0.0536 & 0.0550 & 0.0850 & 0.0813 \\
$\mathbf{H}_{2} \mathbf{O}_{2}$ (g) & $4.29(10 \%)$ & $4.281(10 \%)$ & $6.924(5 \%)$ & $8.175(5 \%)$ \\
\hline
\end{tabular}

Analysis:

Methylene Chloride was used as extraction solvent for the products. About 1:1 volume of methylene chloride was added for extraction of reaction products. The analysis of this extraction sample was done by GC/MS. A sample GC chromatogram is attached for each test.

For test 1, which was carried out at the highest temperature $\left(400^{\circ} \mathrm{C}\right)$ and with longest reaction time (30 min), GC chromatogram showed that very little non-volatile products in the solvent. Test 2 was carried out also at $400{ }^{\circ} \mathrm{C}$, but with 15 minutes of reaction time. Two major were shown at 213 and 227 respectively. By mass spectrograph analysis, the most possible product name at 213 peak is either $\mathrm{C}_{5} \mathrm{H}_{7} \mathrm{NO}_{2}$ (2,5 - pyrrolidinedione, 1-methyl-) or $\mathrm{C} 7 \mathrm{H} 12 \mathrm{O} 3$ is. For the peak at $227, \mathrm{C}_{8} \mathrm{H}_{13} \mathrm{NO}_{2}$ or $\mathrm{C}_{8} \mathrm{H}_{18} \mathrm{~N}_{2}$ were suggested by a computer-based finger printing, but the possibility of confirmation were quite low in both cases $(59 \%)$. It is also noted that the concentration of former is much higher than the latter. A few other peaks of this run were identified as the products derived form silicon oil used in the screw of the reactor. The silicon oil was not used for tests 3 and 4 . 
Test 3 was ran at $350^{\circ} \mathrm{C}$ for $30 \mathrm{~min}$. The number of products has sharply increased for this run to more than 20. The computer finger printing suggested that the possible products of this run include: $\mathrm{C}_{6} \mathrm{H}_{14} \mathrm{O}\left(2,3\right.$-dimethyl-1-butanol) at peak $17, \mathrm{C}_{6} \mathrm{H}_{6} \mathrm{O}$ (phenol) at peak 114, $\mathrm{C}_{5} \mathrm{H}_{7} \mathrm{NO}_{2}$ (1-methyl-2,5 pyrrolidinedione) or $\mathrm{C}_{7} \mathrm{H}_{12} \mathrm{O}_{3}$ at peak $198, \mathrm{C}_{14} \mathrm{H}_{20} \mathrm{O}_{2}$ at peak $573, \mathrm{C}_{15} \mathrm{H}_{16} \mathrm{O}$ at peak 830 , alkanes such as $\mathrm{C}_{9} \mathrm{H}_{20}$ and $\mathrm{C}_{12} \mathrm{H}_{26}$, and $\mathrm{C}_{18} \mathrm{H}_{20}$ (2,4-diphenyl-4-methyl-pentene).

Test 4 was ran at $300^{\circ} \mathrm{C}$ for $30 \mathrm{~min}$. There were much less products in this run that in test 3 . The main products are: $\mathrm{C}_{6} \mathrm{H}_{7} \mathrm{NO}$ (1-methyl 1H-pyrrole-2-carboxaldehyde), $\mathrm{C}_{8} \mathrm{H}_{18}$ (3-Ethyl-2-methyl-pentane) or $\mathrm{C}_{9} \mathrm{H}_{20}$ (3,4-dimethyl-heptane), and $\mathrm{C}_{15} \mathrm{H}_{16} \mathrm{O}$ (2,3-dimethyl-1-butanol or 2-methyl-1-pentanol). The full sketch of the products are shown in Table 2 . 
Table 2. Main Products in the solvent

\begin{tabular}{|c|c|c|c|}
\hline $\begin{array}{c}\text { Experiment } \\
\text { No. }\end{array}$ & $\begin{array}{l}\text { Peak } \\
\text { at }\end{array}$ & Possible Elements & Possible Product name \\
\hline Test 1 & & No products detected & \\
\hline Test 2 & $\begin{array}{l}213 * \\
227\end{array}$ & $\begin{array}{l}\mathrm{C}_{5} \mathrm{H}_{7} \mathrm{NO}_{2} \text { or } \mathrm{C}_{7} \mathrm{H}_{12} \mathrm{O}_{3} \\
\mathrm{C}_{8} \mathrm{H}_{13} \mathrm{NO}_{2} \text { or } \mathrm{C}_{8} \mathrm{H}_{18} \mathrm{~N}_{2}\end{array}$ & $\begin{array}{l}\text { (1-methyl-2,5-pyrrolidinedione) or }(1)^{* *} \\
\text { (3-ethyl-1, 3-dimethyl-2, 5-pyrrolidinedione) or } \\
\text { (2-propanone, ethyl<1-methylethyl }>\text { hydrazone) }\end{array}$ \\
\hline Test 3 & $\begin{array}{l}17 \\
31 \\
114 * \\
198 * \\
573 \\
837 \\
912 \\
951 \\
1186 \\
1274 \\
1355 \\
1432 \\
1513 \\
\end{array}$ & $\begin{array}{c}\mathrm{C}_{6} \mathrm{H}_{14} \mathrm{O} \\
\mathrm{C}_{9} \mathrm{H}_{20} \\
\mathrm{C}_{6} \mathrm{H}_{6} \mathrm{O} \\
\mathrm{C}_{5} \mathrm{H}_{7} \mathrm{NO}_{2} \text { or } \mathrm{C}_{7} \mathrm{H}_{12} \mathrm{O}_{3} \\
\mathrm{C}_{14} \mathrm{H}_{20} \mathrm{O}_{2} \\
\mathrm{C}_{18} \mathrm{H}_{20} \\
\mathrm{C}_{18} \mathrm{H}_{20} \\
\mathrm{C}_{18} \mathrm{H}_{20} \\
\text { no fit found } \\
\mathrm{C}_{13} \mathrm{H}_{28} \\
\mathrm{C}_{13} \mathrm{H}_{28} \\
\mathrm{C}_{12} \mathrm{H}_{26} \\
\mathrm{C}_{28} \mathrm{H}_{34} \mathrm{O} \\
\end{array}$ & $\begin{array}{l}\text { (2,3-dimethyl-1-butanol) or (2-methyl-1-pentanol) } \\
\text { (2,3,5-thrmethyl-hexane) or (4-methyl-octane) } \\
\text { (phenol) } \\
\text { (1-methyl-2,5-pyrrolidinedione) or (1)** } \\
\text { (2,5-cyclohexadiene-1,4-dione,2,6-bis<1,1- } \\
\text { dimethylethyl }>\text {-) } \\
\text { (2,4-diphenyl-4-methyl-2<Z>-pentene) } \\
\text { (2,4-diphenyl-4-methyl-2<E>-pentene) } \\
\text { (2,4-diphenyl-4-methyl-2<E >-pentene) } \\
\text { no fit found } \\
\text { (2,4,6-trimethyl-decane) or (3,7-dimethyle-undecane) } \\
\text { (3,7-dimethyle-undecane) or (3,8-dimethyl-undecane) } \\
\text { (3,8-dimethyl-undecane) or (2,4,6-trimethyl-dodecane) } \\
\text { (2,4-bis<dimethylbenzyl>-6-T-butylphenol }\end{array}$ \\
\hline Test 4 & $\begin{array}{l}20 \\
34 \\
142 * \\
144 *\end{array}$ & $\begin{array}{c}\mathrm{C}_{6} \mathrm{H}_{14} \mathrm{O} \\
\mathrm{C}_{8} \mathrm{H}_{18} \text { or } \mathrm{C}_{9} \mathrm{H}_{20} \\
\mathrm{C}_{6} \mathrm{H}_{7} \mathrm{NO} \\
\mathrm{C}_{6} \mathrm{H}_{7} \mathrm{NO}\end{array}$ & $\begin{array}{l}\text { (2,3-dimethyl-1-butanol) or (2-methyl-1-pentanol) } \\
\text { (3-Ethyl-2-methyl-pentane) or (3,4-dimethyl-heptane) } \\
\text { (1-methyl 1H-pyrrole-2-carboxaldehyde) } \\
\text { (1-methyl 1H-pyrrole-2-carboxaldehyde) }\end{array}$ \\
\hline
\end{tabular}

$(1)^{* *}$ : Full identification unavailable. 


\section{Direction of Future Research}

The results obtained to this point indicate that the proposed scheme of utilizing hydrogen peroxide as the oxidative lignin degradation reagent is a valid concept that warrants further investigation although the results are sketchy at this point. The following are the main items of investigation we think should be pursued if this research is to be explored.

1. Verification of the mechanisms of the oxidation reaction between lignin (biomass-to-ethanol lignin) and hydrogen peroxide.

2. Expand the reaction conditions in terms of temperature, pressure, reagent ratio, and reaction time and identify the products in liquid and gas phase.

3. Evaluate the reaction performance and efficiency of the process under selected reaction conditions.

4. Evaluate the economic factors for the process.

5. Assimilate a process design and integrate into the biomass-to- ethanol process.

\section{Preparation of Lignin Generated during Total Hydrolysis of Yellow Poplar}

In addition to the work described above, a sample quantity of yellow poplar lignin was prepared and sent to Dr. B. Hames of NREL for preliminary test and comparison of properties between this and the one produced from NREL total hydrolysis system.

For lignin production, a medium sized perocolation reactor (3"ID x 12"L) was operated in three stages, $174 \mathrm{C}, 225 \mathrm{C}$, and $235 \mathrm{C}$ with $0.07 \% \mathrm{H}_{2} \mathrm{SO}_{4}$, taking one reactor volume fluid at each stage. The reaction conditions were identical to those applied in the NREL total hydrolysis system with one exception that the spring assisted bed compaction is not applied here. The hydrolysates at each stage were collected separately and stored in a refrigerator overnight. The lignin precipitated during this 
process was filtered and dried. The quantity of lignin generated during the first stage was less than $0.1 \mathrm{~g}$. The lignin samples sent to NREL were the ones generated in the last two stages. 
Attachment:

\section{GC Graph}

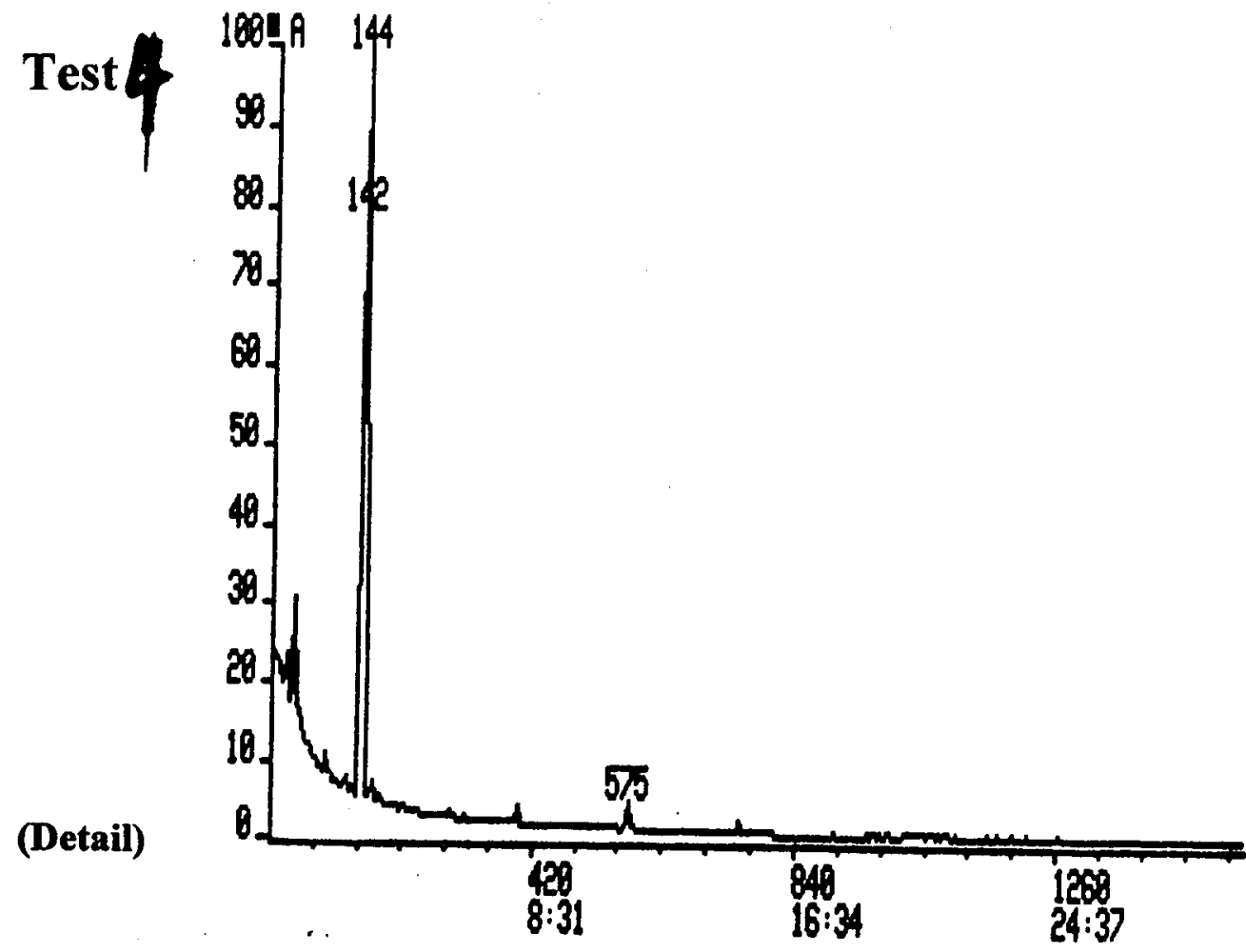

1.68

39917
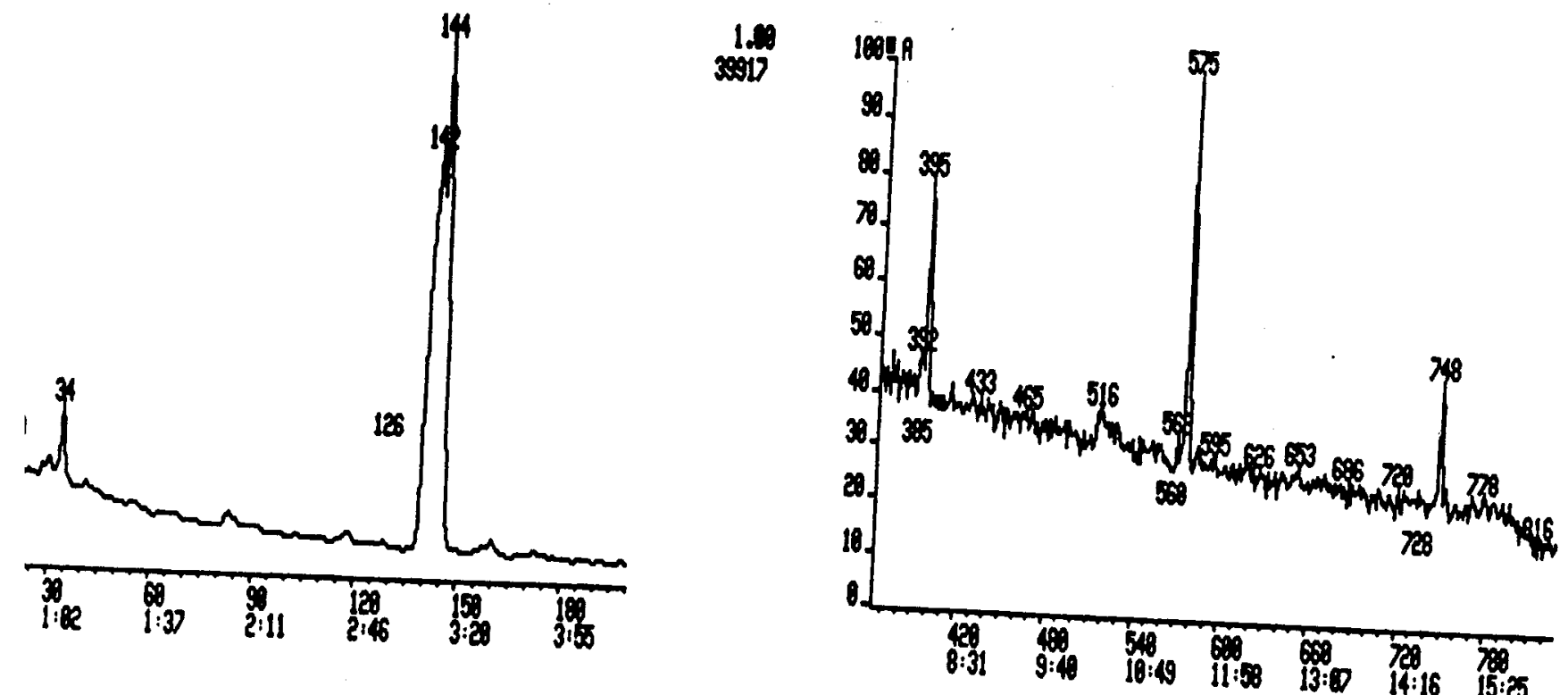
Attachment:

\section{GC Graph}

\section{Test 1}

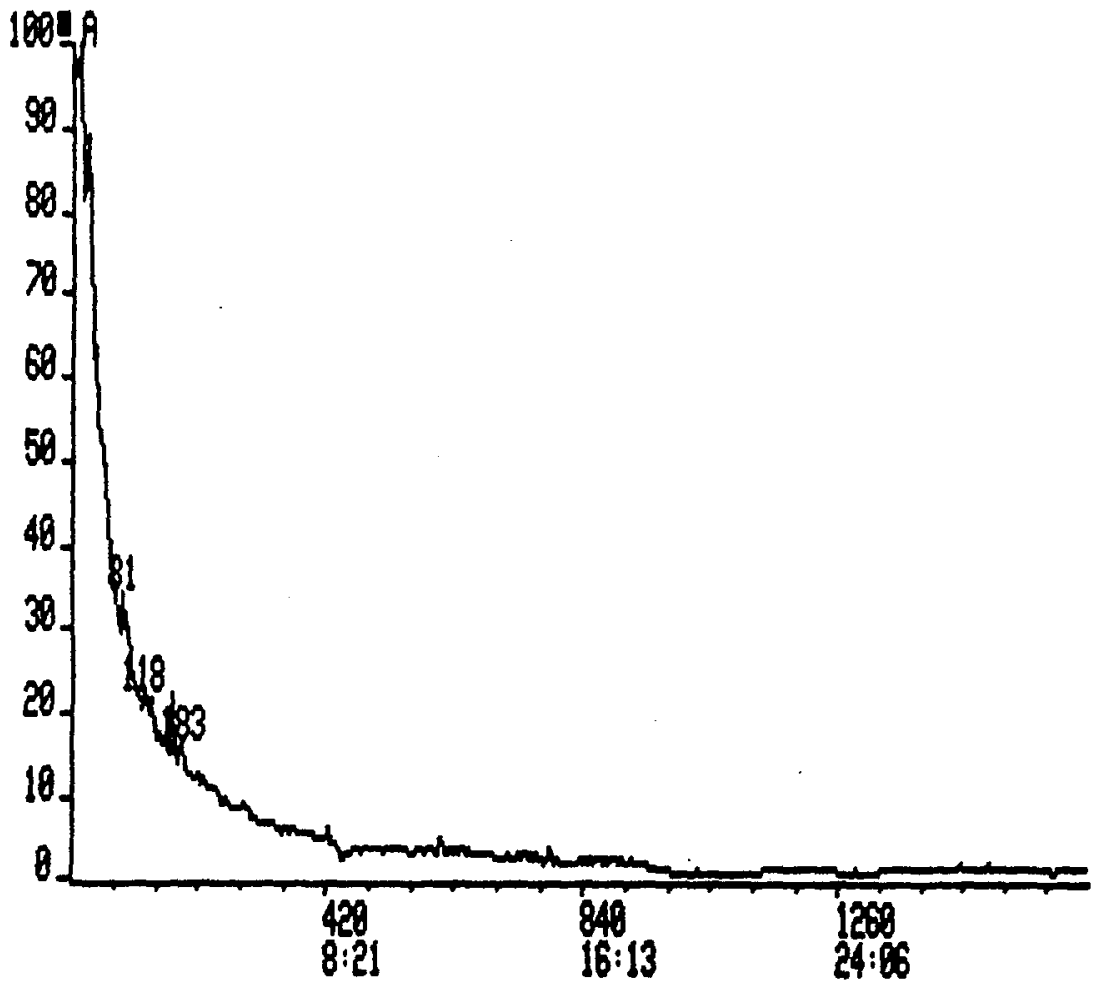

\section{Test 2}

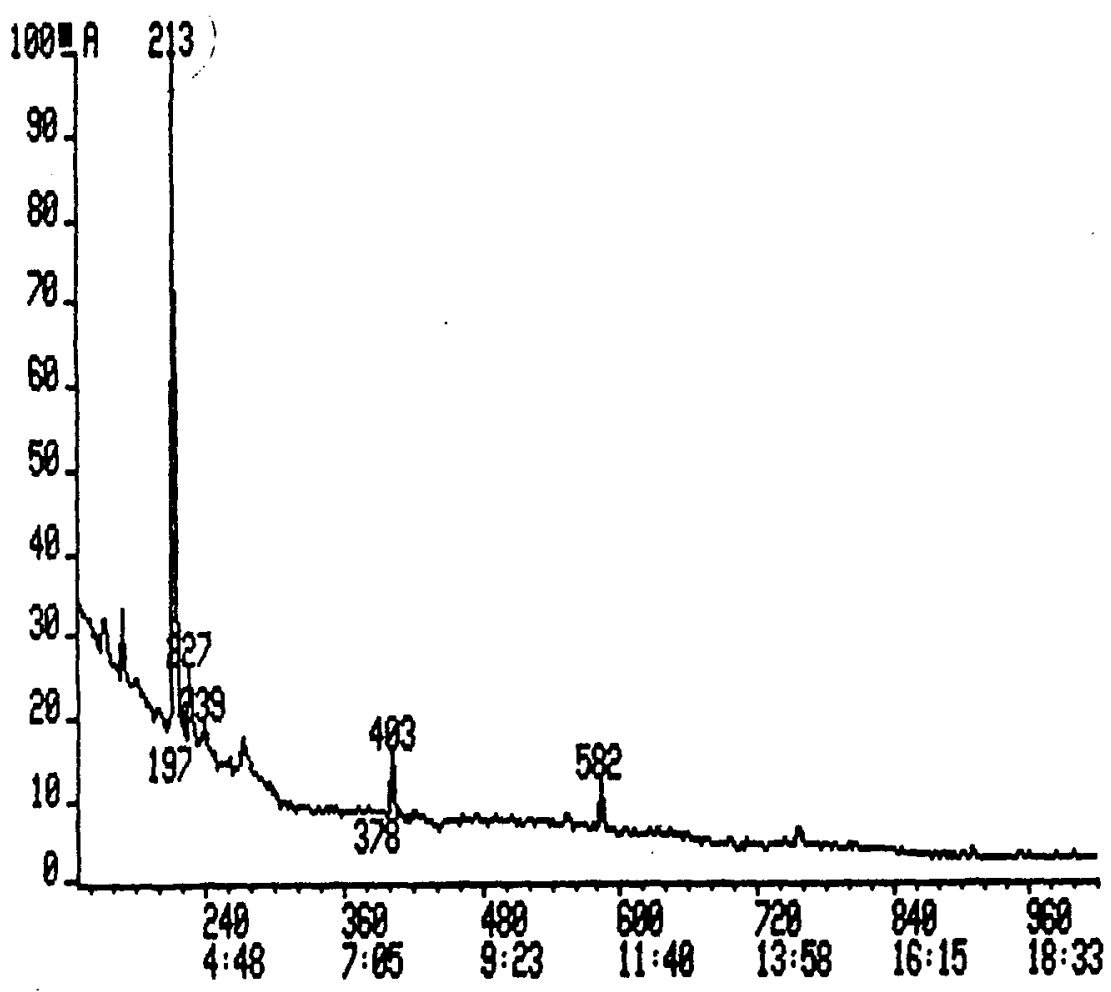


Attachment:

\section{GC Graph}
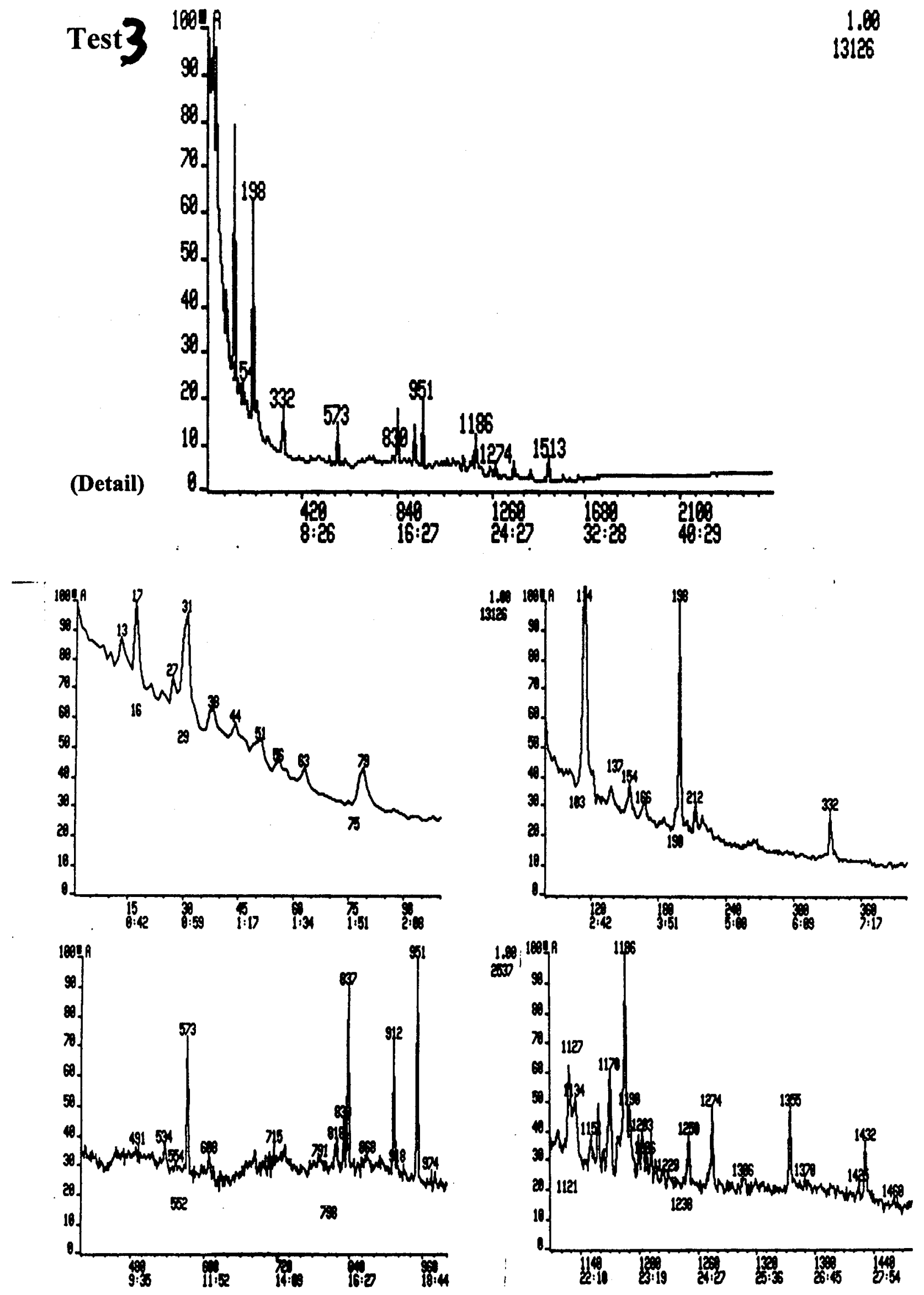

1,00 


\section{Appendix 1}

\section{Fortran Program Source Codes for Modeling of Counter-Current Bed-Shrinking Reactor}

\section{Notes:}

1. This program has been installed into the NREL PC along with the Microsoft Fortran and tested for actual reactor simulation.

2. This program requires 4 subroutines from IMSL library available from Microsoft Professional Fortran program package and external functions defined within the program. 
This program calculates xylose yield in countercurrent shrinking and $c$

nonshrinking bed reactor. The reaction follows pattern: c

$\mathrm{K} 1 \mathrm{k} 2 \mathrm{k} 4$

HC (A) -.->Oligomer (B) -->XYlose (C)-->Decomposition o

Since the uniform temperature, the biphasic nature of hemicellulose is $c$

reflected on the overall $\mathrm{K}, \mathrm{K}=\mathrm{F}^{\star} 1+(1-\mathrm{F}) \mathrm{k} 2$. $\mathrm{C}$

Process varables: tem, u(liquid velocity: $\mathrm{cm} / \mathrm{min}$ ), vo(solid velocity: cm/min) $\mathrm{c}$ acid concentration, $\mathrm{g} / 100 \mathrm{ml}$.

out put data: Z (L/Lo), YA(Ca/CaO), YB(Cb/Cao), YC(CC/CAO)

then yield and sugar concentration can be calculated from YB, YC. C

Yield=epsilon* $(\mathrm{YB}+\mathrm{YC}) *$ omega, here omega $=\mathrm{u} / \mathrm{vo} \quad \mathrm{c}$

when let $f=0.0001$, the process was forced to be a nonshrinking one c

Modified date: $03 / 03 / 98$ by Prashant V Iyer

$\mathrm{C}$

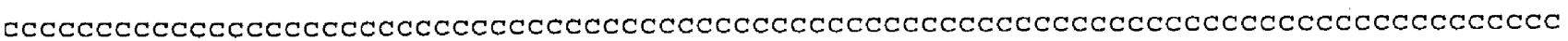

use msimsl

real tem, ac, vo, $u$, bee, coefl, coef2

real lamd, Cao, omega, epsilon

real yield(100), olig(100), xylose (100), qq (100)

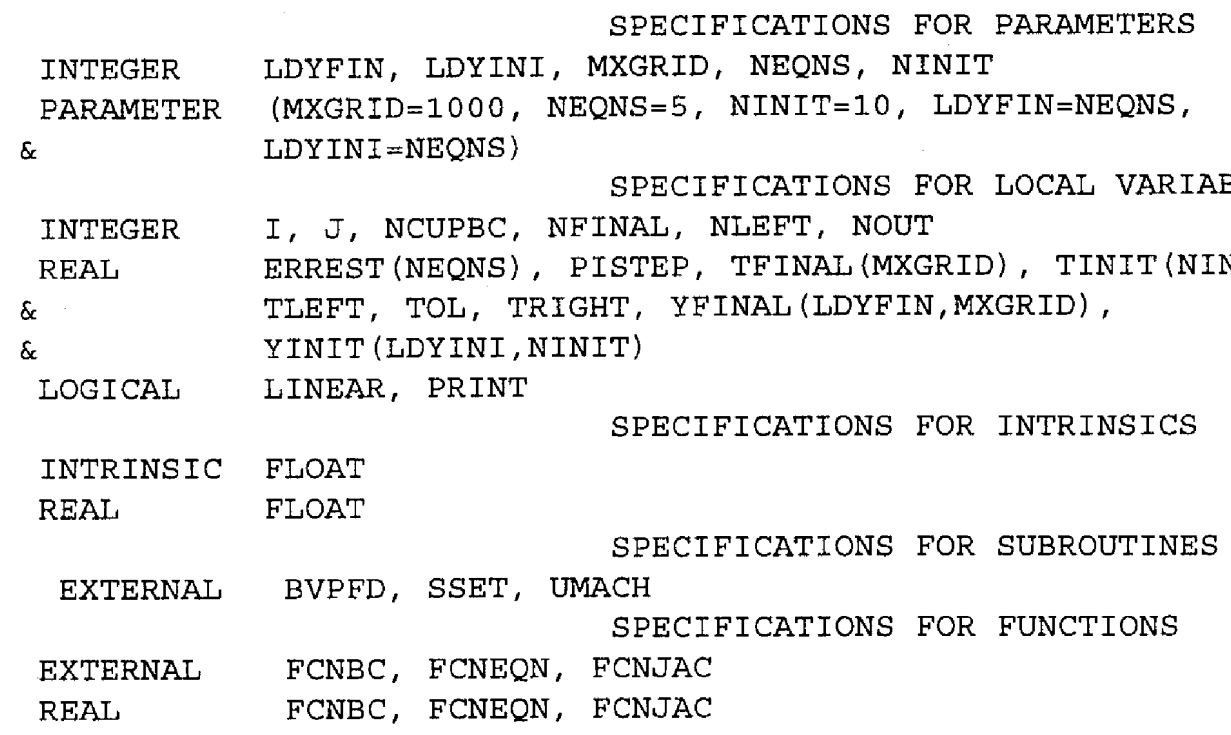

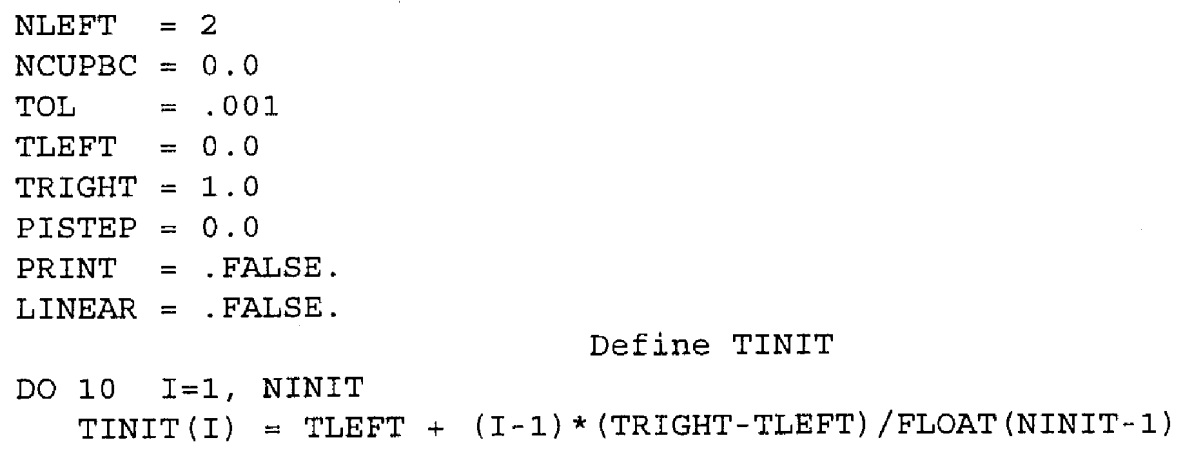


call coeff(tem, ac, vo, u, bee, coef1, coef2,alpha3,alpha4,

\&lamd, Cao, omega, epsilon, f)

Print*, lamd

open (unit $=3, \mathrm{file}=$ "run1. dat", status="old")

write $(3,66)$

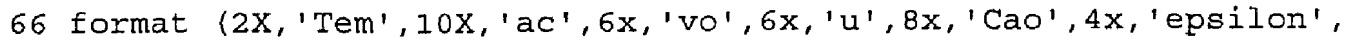
$\& 4 \mathrm{x}, \mathrm{f} \cdot)$

write $(*, 77)$ tem, ac, vo, $u$, Cao, epsilon, $f$

77 format $(2 \mathrm{x}, \mathrm{F} 4.0,7 \mathrm{x}, \mathrm{F} 5.2,3 \mathrm{x}, \mathrm{f} 5.1,2 \mathrm{x}, \mathrm{f5} .1,4 \mathrm{x}, \mathrm{f} 6.2,3 \mathrm{x}$, f5.2,

$\& 5 \mathrm{x}, \pm 5.2\rangle$

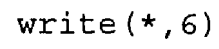

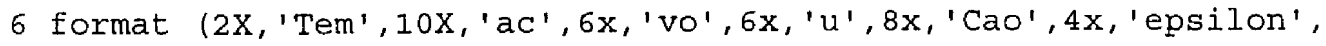
$\& 4 \mathrm{x}$, ' $\mathrm{f}$ ')

write $(3,7)$ tem, ac, vo, $u$, Cao, epsilon, $f$

7 format $(2 \mathrm{x}, \mathrm{F} 4.0,7 \mathrm{x}, \mathrm{F5} .2,3 \mathrm{x}, \mathrm{f5} .1,2 \mathrm{x}, \mathrm{f5} .1,4 \mathrm{x}, \mathrm{f6.2}, \mathrm{xx}, \mathrm{f5.2}$, $\& 5 x, f 5.2)$

Print*, ' $\mathrm{T}=1$, tem, ' $\mathrm{ac}=1, \mathrm{ac}, \mathrm{vo}=1, \mathrm{vo}, \mathrm{\prime} \mathrm{u}=\mathrm{\prime}, \mathrm{v}$

print*, Cao, lamd, luo solve problem

CALL BVPFD 〈FCNEQN, FCNJAC, FCNBC, FCNEQN, FCNBC, NEQNS, NLEFT, \& NCUPBC, TLEFT, TRIGHT, PISTEP, TOL, NINIT, TINIT,

\& YINIT, LDYINI, LINEAR, PRINT, MXGRID, NFINAL,

\& TFINAL, YFINAL, LDYFIN, ERREST)

Calculation and Printing results

write $(*, 5)$

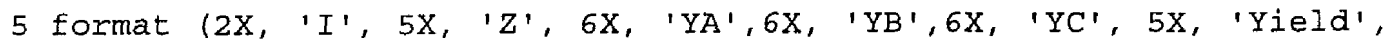
\& $3 \mathrm{x}$, 'Oligomer', $3 \mathrm{x}$, 'Xylose', $3 \mathrm{x}, \mathrm{x} / \mathrm{vo}$ ')

write $(3,55)$

55 format $\left(2 X, ' I ', 5 X, ' Z^{\prime}, 6 X, ' Y A ', 6 X, X^{\prime}, 6 X\right.$, 'YC', 5X, 'Yield',

$\& 3 x$, 'Oligomer', $3 \mathrm{x}$, 'Xylose', $3 \mathrm{x}, \mathrm{\prime} \mathrm{v} / \mathrm{vo}$ ')

do $555 \mathrm{~m}=1$, nfinal.

yield $(m)=(y f i n a l(2, m)+y f i n a l(4, m)) *$ omega*epsilon

olig $(m)=$ Cao*yfinal $(2, m)$

xylose $(m)=$ Cao*yfinal $(4, m)$

$q q(m)=(1$ amd -1.$) /($ lamd-yfinal $(1, m))$

write $(*, 44) m$, tfinal $(m), y f i n a l(1, m), y f i n a l(2, m), y f i n a l(4, m)$,

\& yield $(m), \operatorname{olig}(m)$, xylose $(m), q q(m)$

write $(3,44) m$, tfinal $(m), y f i n a l(1, m), y f i n a l(2, m), y f i n a l(4, m)$,

\& yield $(m)$, olig $(m), x y l o s e(m), q q(m)$

44 format (2X, I1, 2X, F5.2, 2X, F6.3,2X, F6.3,2X, F6.3, 2X, F7.4,

$\& 2 \mathrm{X}, \mathrm{F7} .3,2 \mathrm{X}, \mathrm{F} 7.3,3 \mathrm{X}, \mathrm{F} 6.3)$

555 continue

print*, yfinal $(1,1)$

end

END

SUBROUTINE FCNEQN (NEQNS, T, Y, P, DYDX)

SPECIFICATIONS FOR ARGUMENTS 


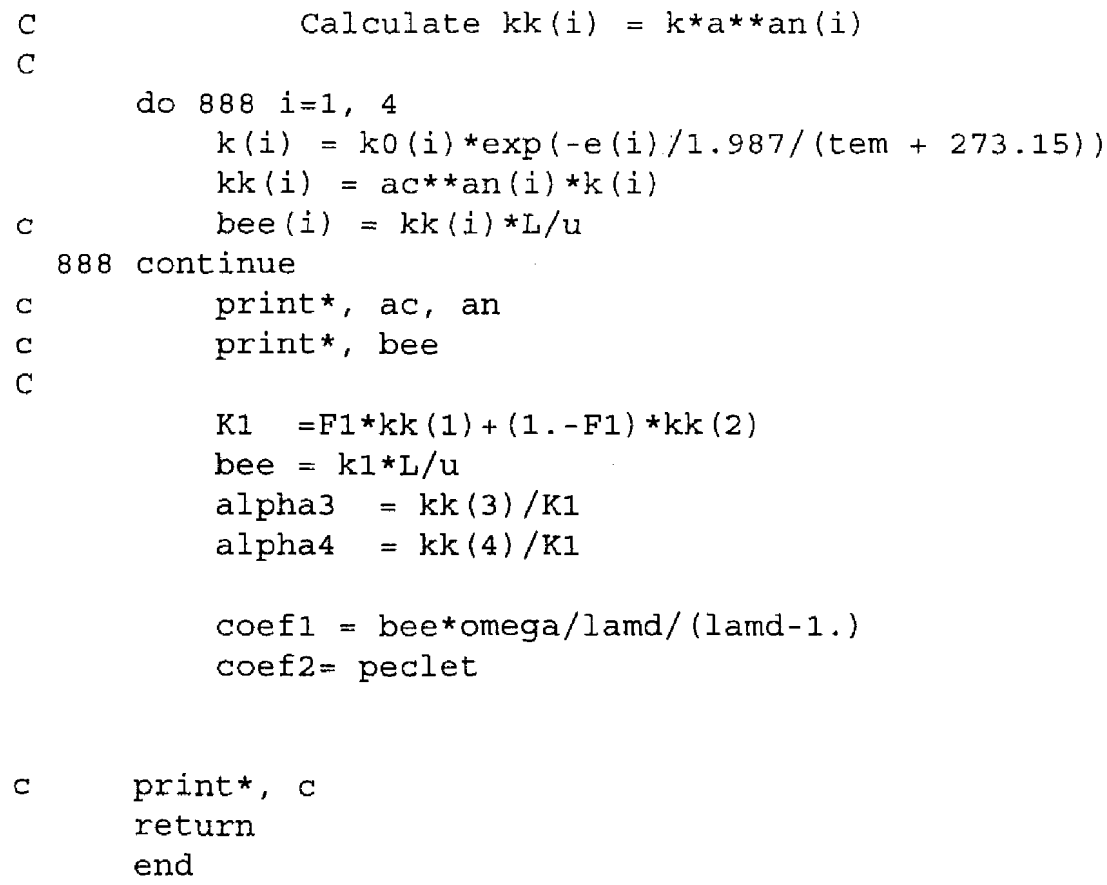




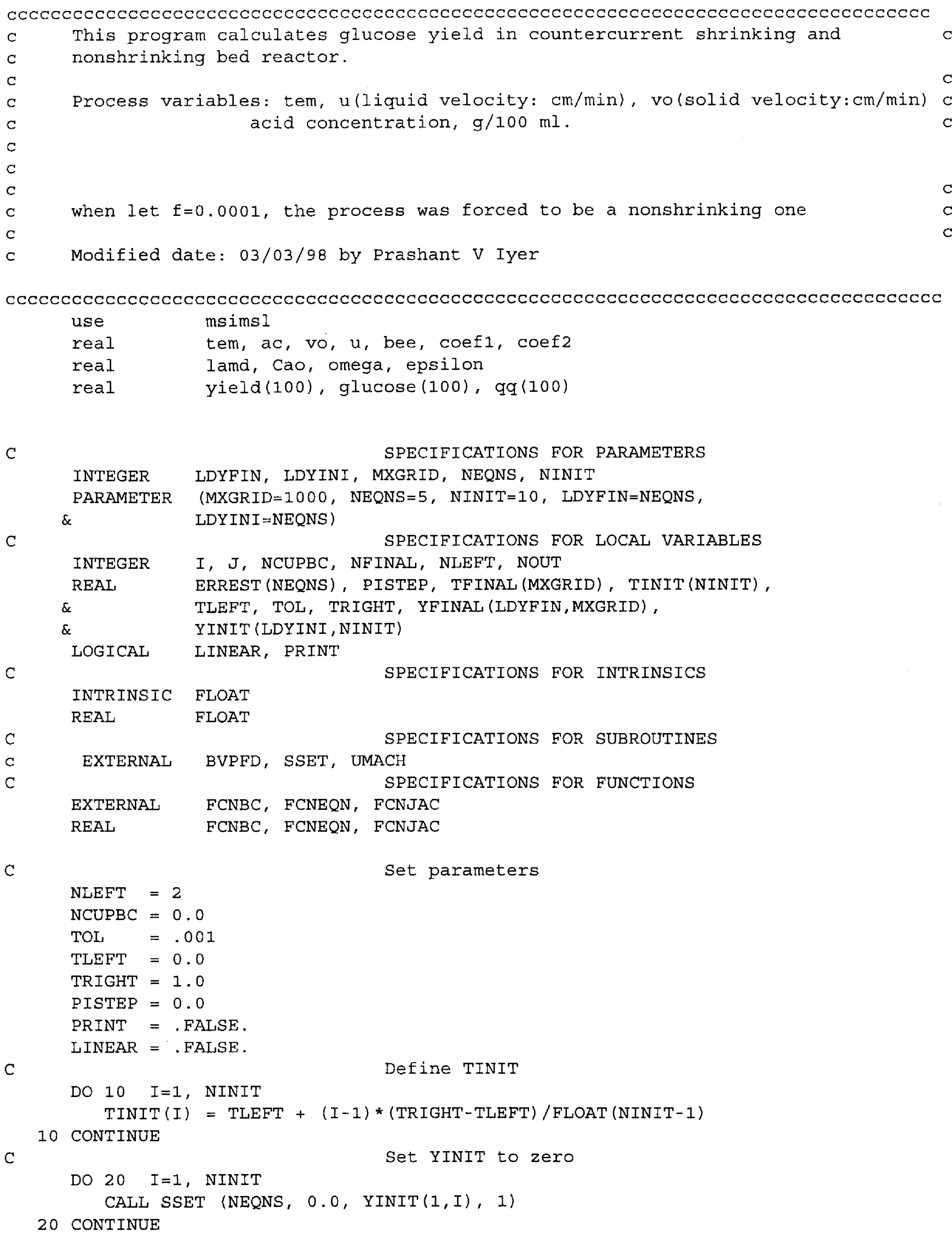

call coeff(tem, ac, vo, u, bee, coef1, coef2,alpha3,alpha4, \&lamd, Cao, omega, epsilon, f) 


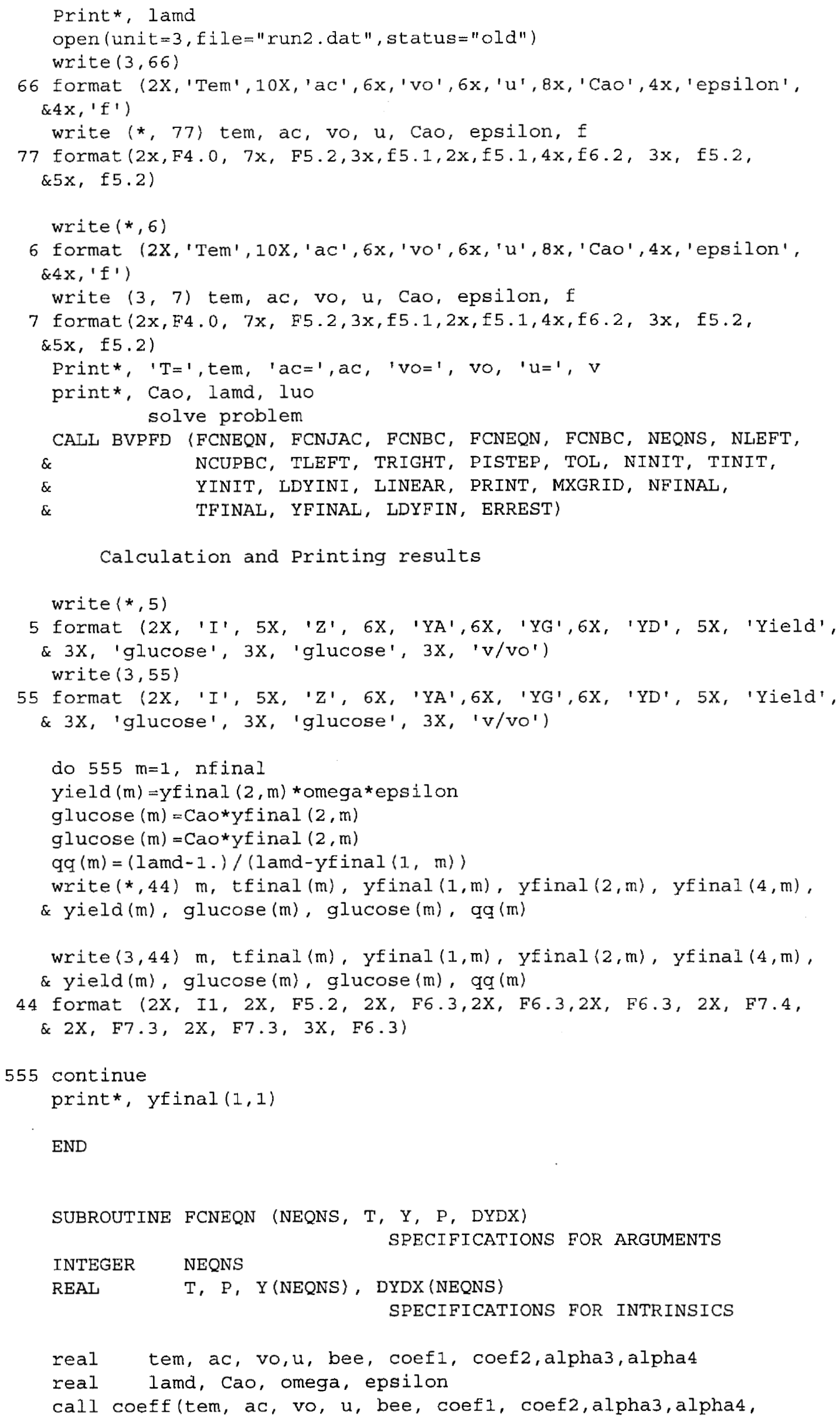




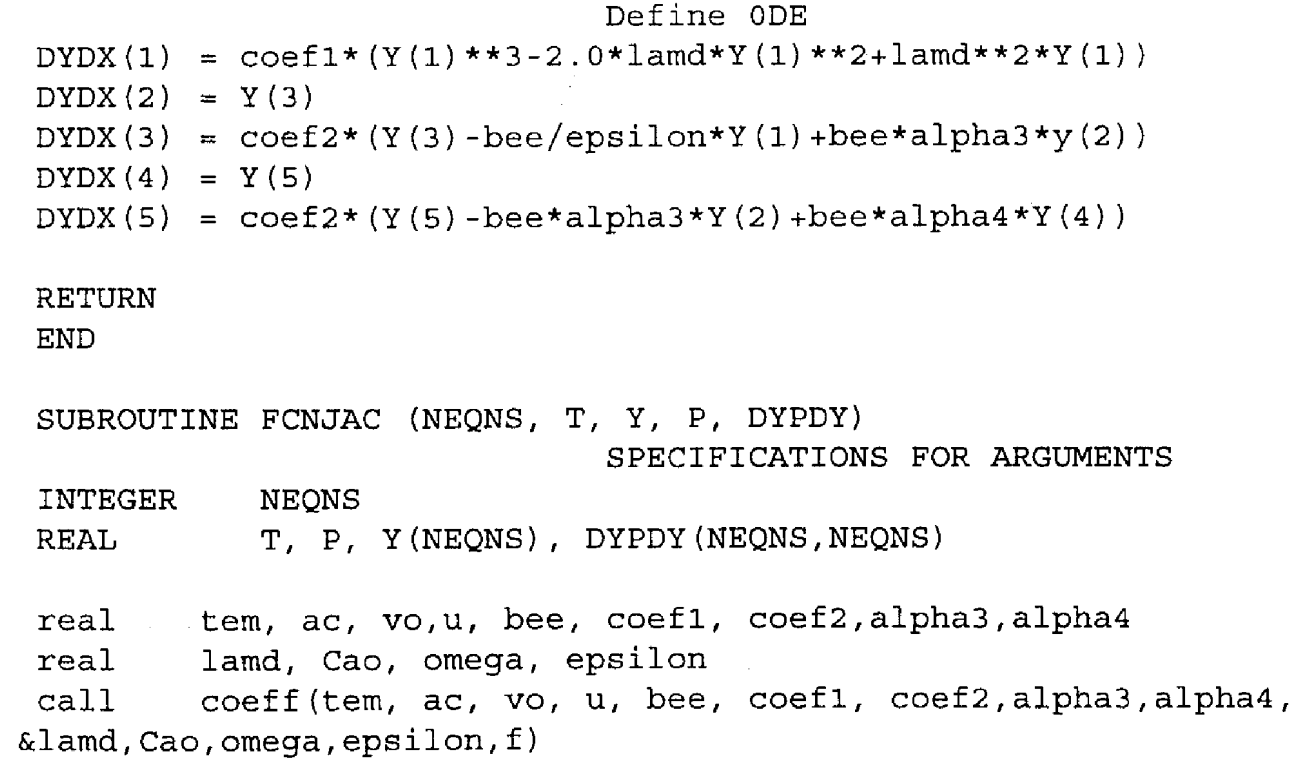


$F(2)=Y L E F T(4)$

$F(3)=\operatorname{YRIGHT}(1)-1.0$

$F(4)=$ YRIGHT (3)

$\mathrm{F}(5)=$ YRIGHT (5)

RETURN

END

subroutine coeff(tem, ac, vo,u,bee, coef1, coef2,alpha3,alpha4, \&lamd, Cao, omega, epsilon, f)

real tem, ac, $u$, vo, $L, f, H o, F I, l u o$, Cao

real omega,epsilon alpha3, alpha4, peclet

real bee, coefl, coef $2, \operatorname{coef} 3$, lamd

real $\mathrm{K} 1$, an $(4), \mathrm{kO}(4), \mathrm{e}(4), \mathrm{k}(4), \mathrm{kk}(4)$

Input Initial data

unit: vo, $u=\mathrm{cm} / \mathrm{min} ; \mathrm{luo}=\mathrm{g} / \mathrm{ml}, \mathrm{HC}=\mathrm{g} / 100 \mathrm{~g}, \mathrm{CaO}=\mathrm{g} / 100 \mathrm{~mL}$

Tem $=225$

ac $=0.08$

$\mathrm{u}=3.5$

vo $=0.505$

Print*, tem, ac, $u$, vo

$\mathrm{f} \quad=0.001$

epsilon $=0.84$

I. $=15.24$

HO $=85$.

$\mathrm{F} 1=0.65$

luo $=1.0$

Cao $=$ luo*Ho* (1.- epsilon)

omega $=u / v o$

lamd $\quad=(1 .-$ epsilon $) *$ luo $* 100 /(f *$ Cao $)$

peclet $=6.25$

Input kinetic data

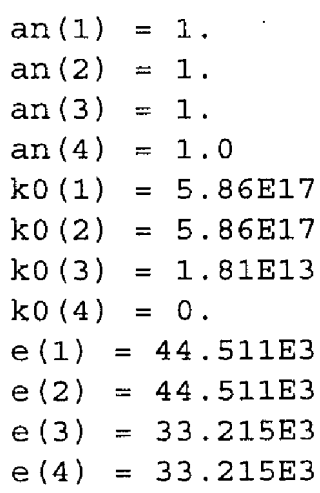




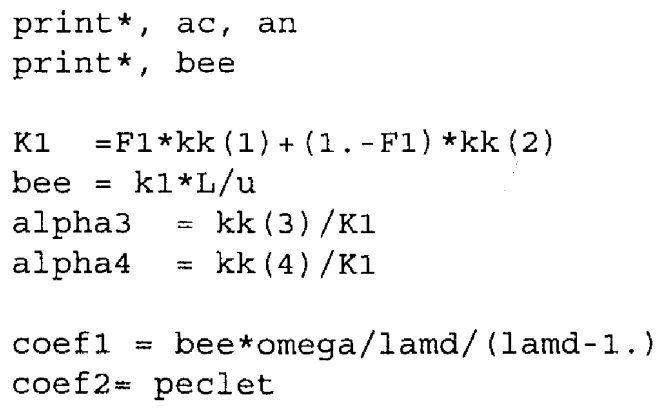

return

end 


\section{Appendix 2}

\section{List of Papers Published or Submitted for Publication}

\section{Full or partial financial support from NREL is acknowledged for the following papers.}

\section{Published-}

1. Rongfu Chen and Y. Y. Lee, "Shrinking -Bed Model for Percolation Process Applied to Dilute-Acid Pretreatment/Hydrolysis of Cellulosic Biomass", Applied Biochem. \& Biotechnol., Vol. 70-72, 37-50, 1998

2. Zhangwen Wu and Y. Y. Lee, "Non-isothermal Simultaneous Saccharification and Fermentation for Direct Conversion of Lignocellulosic Biomass to Ethanol", Applied Biochem. \& Biotechnol, 70-72, 479-492, 1998

3. Zhangwen Wu and Y. Y. Lee, "Effect of Ethanol on Enzymatic Hydrolysis of Cellulose", Biotechnology Letters, Vol.9, No.10, 977-979, 1997,

\section{*Submitted-}

1. Zhangwen Wu, Y. Y. Lee, and R. W. Torget, "Modeling of Countercurrent Continual Shrinking-Bed Reactor System Applied to theDilute-Acid Hydrolysis of Lignocellulosic Biomass", Submitted for publication in Biotechnol Bioresource Technology

2. Prashant Iyer and Y. Y. Lee, "Simultaneous Fermentation and Extractive Fermentation of Lignocellulosic Materials into Lactic Acid in Two-Zone FermenterExtractor System", Submitted for publication in Applied Biochem. \& Biotechnol Technology,

*Abstracts are attached. 


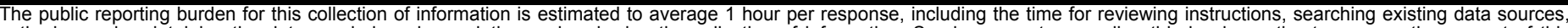

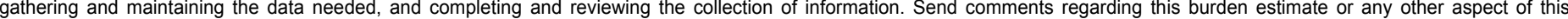

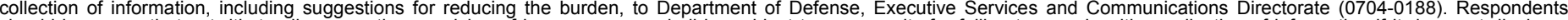

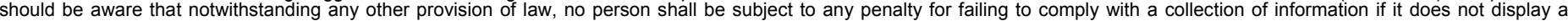
currently valid OMB control number.

PLEASE DO NOT RETURN YOUR FORM TO THE ABOVE ORGANIZATION.

\section{REPORT DATE (DD-MM-YYYY) August 2004 \\ 2. REPORT TYPE \\ Subcontract Report}

4. TITLE AND SUBTITLE

Kinetic and Modeling Investigation to Provide Design Guidelines

for the NREL Dilute Acid Process Aimed at Total

Hydrolysis/Fractionation of Lignocellulosic Biomass: July 1998

6. AUTHOR(S)

Y.Y. Lee, P. Iyer, Q. Xiang, and J. Hayes
3. DATES COVERED (From - To)

July 1998

5a. CONTRACT NUMBER

DE-AC36-99-GO10337

5c. PROGRAM ELEMENT NUMBER

5d. PROJECT NUMBER

NREL/SR-510-36392

5e. TASK NUMBER

BB04.7610

5f. WORK UNIT NUMBER

\section{PERFORMING ORGANIZATION NAME(S) AND ADDRESS(ES)}

Department of Chemical Engineering

Auburn University

8. PERFORMING ORGANIZATION REPORT NUMBER

RCG-7-17041-01

Auburn, Alabama 36849

9. SPONSORING/MONITORING AGENCY NAME(S) AND ADDRESS(ES)

National Renewable Energy Laboratory

1617 Cole Blvd.

Golden, CO 80401-3393

10. SPONSOR/MONITOR'S ACRONYM(S) NREL

11. SPONSORING/MONITORING AGENCY REPORT NUMBER NREL/SR-510-36392

\section{DISTRIBUTION AVAILABILITY STATEMENT}

National Technical Information Service

U.S. Department of Commerce

5285 Port Royal Road

Springfield, VA 22161

\section{SUPPLEMENTARY NOTES}

NREL Technical Monitor: R. Torget

\section{ABSTRACT (Maximum 200 Words)}

Following up on previous work, subcontractor investigated three aspects of using NREL "pretreatment" technology for total hydrolysis (cellulose as well as hemicellulose) of biomass. Whereas historic hydrolysis of biomass used either dilute acid or concentrated acid technology for hydrolysis of both hemicellulose and cellulose, NREL has been pursuing very dilute acid hydrolysis of hemicellulose followed by enzymatic hydrolysis of cellulose. NREL's countercurrent shrinking-bed reactor design for hemicellulose hydrolysis (pretreatment) has, however, shown promise for total hydrolysis. For the first task, subcontractor developed a mathematical model of the countercurrent shrinking bed reactor operation and, using yellow poplar sawdust as a feedstock, analyzed the effect of: initial solid feeding rate, temperature, acid concentration, acid flow rate, Peclet number (a measure of backmixing in liquid flow), and bed shrinking. For the second task, subcontractor used laboratory trials, with yellow poplar sawdust and 0.07 wt $\%$ sulfuric acid at various temperatures, to verify the hydrolysis of cellulose to glucose (desired) and decomposition of glucose (undesired) and determine appropriate parameters for use in kinetic models. Unlike cellulose and hemicellulose, lignins, the third major component of biomass, are not carbohydrates that can be broken down into component sugars. They are, however, aromatic complex amorphous phenolic polymers that can likely be converted into low-molecular weight compounds suitable for production of fuels and chemicals. Oxidative degradation is one pathway for such conversion and hydrogen peroxide would be an attractive reagent for this, as it would leave no residuals. For the third task, subcontractor reacted lignin with hydrogen peroxide under various conditions and analyzed the resulting product mix.

\section{SUBJECT TERMS}

biofuels; ethanol; fuels; chemicals; pretreatment; shrinking bed; feedstock; biomass; cellulose; hemicellulose; lignin; hydrolysis

\begin{tabular}{|c|c|c|c|c|}
\hline 16. SECURITY & CLASSIFICATI & N OF: & 17. LIMITATION & 18. NUMBER \\
\hline $\begin{array}{l}\text { a. REPORT } \\
\text { Unclassified }\end{array}$ & $\begin{array}{l}\text { b. ABSTRACT } \\
\text { Unclassified }\end{array}$ & $\begin{array}{l}\text { c. THIS PAGE } \\
\text { Unclassified }\end{array}$ & $\begin{array}{l}\text { OF ABSTRACT } \\
\text { UL }\end{array}$ & \\
\hline
\end{tabular}

\section{9a. NAME OF RESPONSIBLE PERSON}

19b. TELEPONE NUMBER (Include area code) 